\title{
Models and Measurements for the Transmission of Submicron-Width Waveguide Bends Defined in Two-Dimensional Photonic Crystals
}

\author{
Henri Benisty, Ségolène Olivier, Claude Weisbuch, Mario Agio, Maria Kafesaki, Costas M. Soukoulis, \\ Min Qiu, Member, IEEE, Marcin Swillo, Anders Karlsson, Bozena Jaskorzynska, Anne Talneau, Jürgen Moosburger, \\ Martin Kamp, Alfred Forchel, Rolando Ferrini, Romuald Houdré, and Ursula Oesterle
}

\begin{abstract}
One of the essential building-blocks of miniature photonic crystal (PC)-based photonic integrated circuits (PICs) is the sharp bend. Our group has focused on the 2-D photonic crystal based on a triangular lattice of holes perforating a standard heterostructure. The latter, GaAlAs-based or InP-based, is vertically a monomode waveguide. We consider essentially one or two $60^{\circ}$ bends defined by one to five missing rows, spanning both cases of monomode and multimode channel waveguides. From intensive modeling and various experimental measurements (both on GaAs and InP), we point out the origin of the present level of bend insertion losses and discuss the merits of the many roads open for improved design.
\end{abstract}

Index Terms-Integrated optics, photonic crystals (PCs), waveguides.

\section{INTRODUCTION}

$\mathbf{T}$ HE POTENTIAL of 2-D photonic crystals (PCs) for miniature photonic integrated circuits (PICs) is a topic under intense investigation. Channel waveguides designed in photonic crystals and operated at frequencies within the photonic bandgap (PBG) are expected to provide waveguiding with low losses and to allow sharp bends [1]-[3]. The preferred photonic crystals that are being investigated are a triangular array of holes perforated through an heterostructure ensuring vertical index confinement through total internal reflection

Manuscript received August 30, 2001; revised March 28, 2002. This work was supported by the European Union IST Project "PCIC."

H. Benisty, S. Olivier, and C. Weisbuch are with the Laboratoire de Physique de la Matière Condensée, Ecole Polytechnique, UMR 7643 CNRS, 91128 Palaiseau Cedex, France.

M. Agio is with the Dipartimento di Fisica "A. Volta," Università di Pavia, I-27100 Pavia, Italy, and also with Ames Laboratory and Department of Physics and Astronomy, Iowa State University, Ames, IA 50011 USA

M. Kafesaki is with the Research Center of Crete, 71110 Heraklion, Crete, Greece.

C. M. Soukoulis is with the Research Center of Crete, 71110 Heraklion, Crete, Greece, and also with Ames Laboratory and the Department of Physics and Astronomy, Iowa State University, Ames, IA 50011 USA.

M. Qiu, M. Swillo, A. Karlsson, and B. Jaskorzynska are with the Department of Microelectronics and Information Technology, Royal Institute of Technology, 16440 Kista, Sweden.

A. Talneau is with the Laboratoire de Physique et Nanostructures, CNRS, 92225 Bagneux Cedex, France.

J. Moosburger, M. Kamp, and A. Forchel are with Technische Physik, University of Würzburg, 97074 Würzburg, Germany.

R. Ferrini, R. Houdré, and U. Oesterle are with the Institut de Micro et Optoélectronique, Ecole Polytechnique Fédérale de Lausanne, CH-1015 Lausanne, Switzerland.

Publisher Item Identifier S 0018-9197(02)05721-4.
(TIR). Waveguiding in straight channels [4]-[10], and at bends [4], [11]-[13] has been reported, but often in a rather qualitative manner, e.g., through scattered light [12] ([13] and [14] are the first optical quantitative account). Very recently, the spectral analysis of bend transmission has also been reported in conferences [15], [16]. In an actual 2-D PC system with TIR confinement in the third direction, the ultimate system is a monomode channel waveguide used in a regime where out-of-plane losses are as low as possible. These losses are rigorously zero only in straight guides defined in systems such as air-bridge or silica-substrate membranes [17]. Only then can a part of the guided mode dispersion be located below the air (or silica) light line, $\omega=c k_{/ /} / n$ ( $n=1$ for air and about 1.5 for silica). Such rules restrict the bandwidth to a narrow interval with a dispersion that tends to be that of "heavy" photons. Furthermore, as soon as we leave straight guides and consider bends, there is no more $k$ in the system, so that large losses are again likely to occur. For these reasons, and also because of the more generic heterostructure technology associated, we have studied in recent years systems that retain a rather high index cladding [7], [18]-[24]. We have established the main rules that lead to low-loss operation in these systems [25], [26]. One essentially requires a sufficiently deep etching and medium diameter holes (air-filling fractions around 30\%), as well as a modest index step between the core and cladding. This last option, of course, leads to increasing constraints on the etch depth as the mode is rather less confined vertically.

The present authors, organized in a European Union research consortium, have decided to investigate in-depth this second alternative. In this paper, we report in particular on the modeling and characterization efforts that have been undertaken on bends in order to spot the best candidates for miniature systems that are yet easily amenable to integration with other elements (cavities, lasers, combiners, ...) in order to present convincing demonstrations of PC-based photonic integrated circuits.

The rest of the paper is organized as follows. In Section II, we present the various modeling alternatives that have been used to tackle this problem, all based on the finite-difference-time-domain (FDTD) calculation scheme. In Section II-A, the layout of two "calculation setups" and associated field sources that avoid a number of spurious effects is first detailed. Then, in Section II-B, the influence of the PC parameters and of the guide width are discussed. The effect of the bend design itself is studied in some detail in Section II-C, and simple and double 
bends are also compared. To conclude Section II, Section II-D introduces phenomenological losses in the air holes, that are well suited to account for 3-D out-of-plane radiation losses of the actual system, as discussed in [25]-[27]. These losses tend to smooth out the calculated spectra by reducing the impact of interferences between the waveguide modes.

In Section III, we describe the results of various kinds of measurements using the designs investigated in Section II. In Section III-A, we present measurements obtained by an end-fire method on InP systems, etched by a methane-based reactive-ion-etching (RIE) process. In this case, light from a tunable laser source is launched into the heterostructure through adequate optical fibers. The issue of light polarization is addressed in some detail. Of particular interest in this heterostructure is the possibility of fabricating and investigating access ridges that closely parallel those used in the numerical simulations. In Section III-B, we turn to experiments performed in GaAlAs heterostructures by means of an internal source, using the photoluminescence of self-organized InAs quantum dots as an internal probe, as amply reported elsewhere [19], [20], [22]. In this case, the PC was etched by electron-cyclotron-resonance reactive ion etching (ECR-RIE).

In Section IV, we compare the results and simulations with two issues in mind: 1) we want to sort out what part of a bend insertion loss comes intrinsically from the design, and what part is added by the (pseudo-) radiation losses and 2) what are the perspectives for improving the performances of the existing designs.

\section{Modeling OF PC BendS}

\section{A. Principle of Calculation and Layout Suited to Transmission Measurement}

The modeling of guides based on the 2-D photonic crystal has been based on the supercell method in the early days [28]. Of course, this method only gives eigenmodes end eigenfrequencies, and is not suited to understanding the transmission properties of such waveguides. Among the various available electromagnetic methods used in the field of photonic crystals (Transmission-Matrix-Methods [29], Sakoda's method [30], etc...), the FDTD method has become the method of choice to tackle such problems, due to its versatility, tractability, and acceptable computational cost (at least in two dimensions) [31], [32]. By means of the time-domain method, one can obtain spectral characteristics by performing a single simulation followed by a Fourier transform, whereas the frequency domain methods require a separate simulation for each frequency. This is documented in [33]-[35]. In-plane 2-D simulations are performed using the effective index $n_{\mathrm{eff}}$ of the underlying slab waveguide to account for the third dimension. Of course, it does not lend itself in a straightforward manner to a modal analysis, but this option is still possible by adequate projection of the simulation results. Actually, the inherent selectivity of a genuine waveguide translates into a requirement for parasitic fields generated by an unsuited field source not to interfere with the fields that have, indeed, propagated through the guide. This is accomplished by taking care that the best possible absorbing conditions are set up, the so-called perfectly matched layers (PMLs) [36], and also by setting up a source that fits well into the waveguide modes of interest, channeling light inside the waveguide, rather than outside, with the best possible ratio.

Two configurations for the 2-D FDTD simulations may be used. They correspond quite well to the two experimental setups described in Section III. We have checked them on numerous examples and found only minor discrepancies that are easily understood from their different configurations.

The first method, illustrated in Fig. 1(a), is to use only a fine adjustment of the source width and location, at a moderate distance in front of the PC waveguide entrance, but without any extra structures other than this PC waveguide. Unwanted light is then minimal and has no marked spectral features, but we nevertheless cared that it could not significantly reach the exit on account of extremely weak reflections at the PMLs. The input and output detectors can then be safely located close to, but outside, the PC waveguide ends. The exit detector is a segment oriented normally to the exit guide axis, and only the component of the Poynting vector parallel to that axis is stored and integrated. The incident power is measured with the detector in front of the source but without a PC structure to avoid any reflection. The outgoing power is that measured at the detector just after the PC structure. The transmission $T$ is the ratio of output to incident power. The width and position of the source are optimized so that the incident mode has maximum coupling to the PC waveguide mode and the transmission is maximum. This is also what is sought in the experimental conditions with an internal source. Also, this condition removes the ambiguity that could come from a large power reflected at the entrance for large sources, for example. We discuss later the issue of obtaining the bend transmission by ratioing the output power of a bend to that of a straight guide.

This is not the only possible method. Another typical layout for 2-D FDTD simulation of straight guide transmission is shown in Fig. 1(b). The system consists of the PC waveguide placed between two "ridge access waveguides" (a dielectric ridge with air as the outer medium). These access waveguides allow guided waves to be safely injected into the PC guide that have no chance to interfere with the output. These access guides also may naturally feed a well-defined mode into the PC waveguide (the fundamental mode of the ridge waveguide), and efficiently collect the output power in the same mode as well. The field source then lies along a segment intersecting the input guide. Its spatial profile is adjusted to the guided mode of the pseudoridge, while its temporal profile is a Gaussian pulse centered around the frequency of interest, e.g., the photonic gap central frequency. Fig. 1(e) shows the adaptation of this numerical setup to the case of a $60^{\circ}$ bent $\mathrm{PC}$ waveguide.

Let us briefly comment on the propagation constants of the modes involved, either in the $\mathrm{PC}$ waveguide or in the ridge waveguide. In three dimensions, the mode effective index of a ridge guide is lower than that of the supporting planar waveguide due to the extra horizontal confinement. Of course, we deal with guides that are monomode vertically. When using the planar mode effective index in the 2-D simulation, the resulting ridge mode index will be close to the exact one unless one approaches the case of narrow ridge guides, with widths less than twice the height, roughly. However, this error due to the 2-D projection 


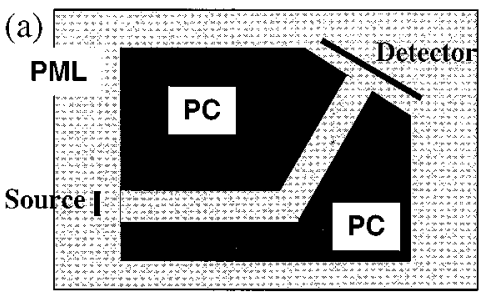

(b)

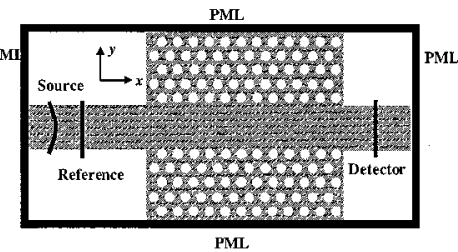

(e)

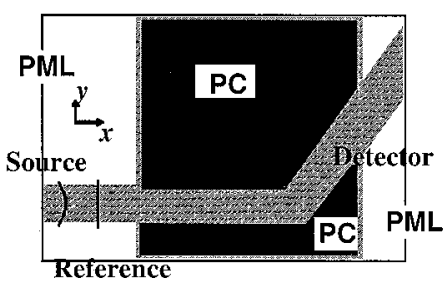

(c)

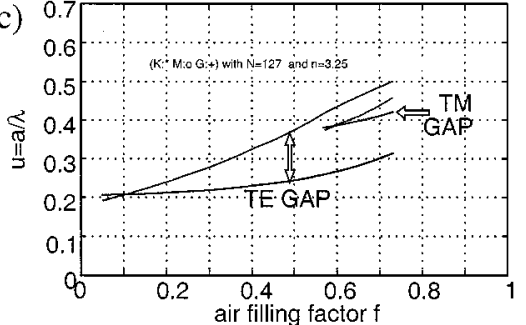

(d)

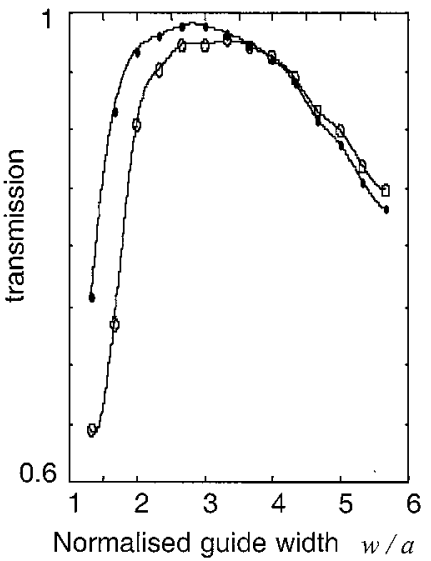

Fig. 1. (a) Layout for the FDTD modeling of the transmission through a single bent PC waveguide. (b) Layout for the FDTD modeling of the transmission through a straight PC waveguide with ridge access wavguides. (c) Gap map in two dimensions for a triangular lattice of air holes in a dielectric matrix of refractive index $n=3.25$. (d) Numerical calculation of ridge-to-PC coupling efficiency as a function of normalized guide width $w / a$, for the case of the three-missing-row guide ("W3"), hence the maximum around $w / a=3$. The open circles are the average transmission for the normalized frequency range 0.22 to 0.25 , while the solid circles are for the normalized frequency range 0.27 to 0.30 . (e) Layout for a $60^{\circ}$ bent PC waveguide with access ridges.

will be essentially the same for the ridge fundamental mode and the PC channel guide fundamental mode, since both modes have similar characteristics when the coupling is optimized. Hence, although we work in 2-D, taking the planar effective index as the "dielectric index," similar in the ridge or in the PC sections, still gives a high accuracy, except maybe for the narrowest guides.

For both methods, too low or too high frequencies that tend to ignore the crystal are, in principle, separated in the final Fourier transform. However it is safer to limit the frequency span of the pulse for two reasons. The first one is that it proves safer to minimize the numerical impact of fields at such unwanted frequencies. The second reason is the fact that the spatial extension of the pulse varies with wavelength $\lambda$, although this is not a major issue with the fundamental mode. The transmission is computed as the ratio of the output and input powers obtained by integration of the Poynting vector fluxes at the detector and the reference lines, respectively. For both systems, the transmission may thus contain a sizable number of higher modes. The issue of the transmission in the fundamental mode is not easy to tackle with the FDTD method. Moreover, we will see that there are various reasons why higher modes are not so large, among which their out-of-plane losses and the collection optics. The proper comparisons between experiments and models are, of course, crucial to assessing this particular aspect.

Finally, the grid (the $d x$ and $d y$ used in the FDTD) and the time steps are similar to those of [31], [37]. Errors were checked to be below the $1 \%$ threshold for typically meshing $20-26$ points per unit cell. The convergence was checked with denser meshes on parts of the data. A rectangular mesh proves useful in the first and second methods to model all the holes the same way in spite of the triangular lattice of the photonic crystal. Furthermore, in the first method, more different $d x$ and $d y$ are also needed to implement the tilted detector. Anyway, note that in the case of a bend not at $90^{\circ}$ and a rectangular mesh, the two arms cannot be modeled with the same symmetry.

Even though most of the following calculations, though, were done with the first method, without access guides, let us insist that one important result of the consortium work is that both methods were compared and gave close results for straight guides and bends.

Let us now turn to the choice of the photonic crystal parameters. As stated earlier, we use as the 2-D material index the effective index of the vertically monomode heterostructure. Its value is around $n_{\mathrm{eff}}=3.25$ for wavelengths around $\lambda=1550 \mathrm{~nm}$, and InP-based planar guides, while it is around $n_{\mathrm{eff}}=3.40$ $\left(\varepsilon_{\mathrm{eff}}=11.56\right)$ around $1000 \mathrm{~nm}$ for GaAs-based planar guides. The main influence of this $5 \%$ difference in the bandstructure is a shift of all features (band edges) toward lower frequencies, proportionally to $1 / n_{\mathrm{eff}}$. For bends, of course, the detailed values of transmission depend on this index, but the trends and all prominent features are barely affected by this difference. For this reason, we focus on either one or the other system, depending on the specific application. For the sake of clarity, Table I gives the parameters used in all the figures presented here.

Fig. 1(c) shows the TE "gap map," i.e., the frequency range of the first TE gap as a function of the air filling factor $f$, for a triangular lattice of air holes in a dielectric matrix of refractive index $n=3.25$. The normalized frequency $u=a / \lambda$ is used, where 
TABLE I

PARAMETERs of Photonic CRystals for the CALCUlations of the Figures Listed in the First Column

\begin{tabular}{|c|c|c|c|c|c|c|}
\hline $\begin{array}{l}\text { Figure } \\
\text { number }\end{array}$ & $\begin{array}{c}\text { dielectric } \\
\text { constant } \\
\varepsilon \\
\text { (real part) }\end{array}$ & $\begin{array}{l}\text { imaginary } \\
\text { part of } \\
\text { dielectric } \\
\text { constant in } \\
\text { holes } \varepsilon^{“}\end{array}$ & $\begin{array}{r}\mathrm{f} \\
\text { (air } \\
\text { filling } \\
\text { factor, \%) }\end{array}$ & $\begin{array}{l}\text { Length of } \\
\text { bend arms } \\
\text { (before, } \\
\text { after) }\end{array}$ & Others & explanation \\
\hline 2 & 11.56 & 0 & 35 & $10 a, 8 a$ & & \\
\hline 3 & 11.56 & 0 & $\begin{array}{l}30,35, \\
40\end{array}$ & $10 a, 8 a$ & & \\
\hline 4 & 11.56 & 0 & 35 & $10 a, 8 a$ & $17 a, 20 a, 16 a$ & \begin{tabular}{l}
\multicolumn{1}{c}{ Length } \\
double \\
straight arms
\end{tabular} \\
\hline 5 & 11.56 & 0 & 35 & $10 a, 8 a$ & & \\
\hline 6 & 11.56 & 0 & 35 & $10 a, 8 a$ & $\begin{array}{l}0.2297(\mathrm{~A}), \\
0.2305(\mathrm{~B}), \\
0.2312 \quad(\mathrm{C})\end{array}$ & $\begin{array}{l}\text { Normalized } \\
\text { frequencies for field } \\
\text { patterns at extrema, } \\
\text { see Fig.5 }\end{array}$ \\
\hline $\begin{array}{l}7 \mathrm{a} \\
7 \mathrm{~b}\end{array}$ & $\begin{array}{l}11.56 \\
11.56\end{array}$ & $\begin{array}{l}0,0.2 \\
0.22\end{array}$ & $\begin{array}{l}35 \\
35\end{array}$ & $\begin{array}{c}9 a, 7 a \\
9 a, 30 a\end{array}$ & & \\
\hline $9 \mathrm{a}$ & 10.56 & 0.22 & 50 & & $\begin{array}{l}18 a, 17 a, 18 a \\
\text { (B) } \\
17 a, 15 a, 17 a \\
\text { (C) }\end{array}$ & \begin{tabular}{l}
\multicolumn{1}{c}{ Length of } \\
double bend \\
straight arms
\end{tabular} \\
\hline
\end{tabular}

$a$ is the lattice period and $\lambda$ the wavelength in vacuum. A sizable gap is obtained around $u=0.22$ as soon as $f=15-20 \%$. Conversely, a TM gap between the second and third bands can coincide with the TE gap at higher air-filling factors, $f=60 \%$ or more. We will typically work in between these extreme cases for the following reasons: small holes tend to be difficult to etch and have an insufficient etch depth, translating into large out-of-plane losses whereas large holes mean also less waveguiding in the third direction with the same kind of consequences.

As for the possibility of investigating radiation losses in the third dimension with FDTD, it requires, in principle, an extremely time consuming full-vector simulation. It has also been shown recently that valuable information could be obtained by means of dissipation introduced in the air holes, at least for PC systems such as those investigated here, with a "conventional" substrate having a moderate index step with respect to the core waveguide, so that all the PBG frequency range lies above the "light-line" of the cladding(s). Numerous configurations (simple crystal rows, cavities, waveguides) have been successfully investigated with this trick and compared more than satisfactorily with experiments [37]. The situation of "membrane" systems, where a part of the PBG lies below the air light-line (or the silica light line) is not so clear, and no experimental/theoretical path toward a simple scheme to quantify losses has been given in these systems yet. For the specific case of a PC waveguide, the propagation losses of the fundamental mode are proportional in a 2-D view to the overlap of the associated electric field with the holes [25]. Small holes are, in principle, more favorable to low-loss propagation. For the higher order modes which rely on Bragg reflection, the propagation losses relate more to the mirror losses of a wave impinging on a plain photonic crystal, for which losses could be more complex than the considerations on the overlap of the electric field with the air holes.

Concerning now the channel waveguides, let us introduce the labeling convention that a $\mathrm{Wn}$ waveguide is a guide consisting of $n$ missing rows in an otherwise perfect triangular PC. The guides with odd $n$ (W1, W3, W5 ...) thus have symmetric boundaries, while those of even $n(\mathrm{~W} 2, \mathrm{~W} 4, \ldots)$ have boundaries shifted by $a / 2$ with respect to each other.

In the FDTD simulation method with access ridges, it is important to avoid reflections between the ridge and the PC sections. Fig. 1(d) shows the coupling efficiency from a ridge (where we recall that only the fundamental mode is excited) 
toward a straight $\mathrm{W} 3 \mathrm{PC}$ waveguide [in the same configuration as in Fig. 1(b)] as a function of the ridge width. It is seen that the coupling efficiency is good (up to 99\%) and that the exact ridge waveguide width is not too critical to achieve good coupling. The fundamental mode is, by far, the only excited in this configuration, be it in the access guide or in the PC guide. Note that such a good coupling is not obvious for guides such as W2, W4, ..., that are not symmetric with respect to their axis: in a pictorial way, the eigenmodes of these guides have distorted wave fronts, leading to a poorer coupling to the straight wave fronts of the access ridge eigenmodes.

In the ridgeless method [Fig. 1(a), with the source close to, but not exactly adjacent to the entrance], the dependence of the coupled power with respect to the width of the impinging wave profile at the guide entrance has a rather more abrupt dependence, but still, the maximum is well defined while not being critical to attain.

The particular nature of PC waveguides makes the quantitative evaluation of the bending efficiency, by referencing a bent waveguide transmission to a straight waveguide transmission, a delicate issue. Namely, unlike conventional guides, the periodic boundaries of a straight PC waveguide may act to prevent transmission in given frequency ranges. A specific phenomenon, that will be referred below, is the so-called mini-stopband (see e.g., [7], [8] for experiments and mode dispersion, and [31] for modeling). This phenomenon is interferometric in nature, just like grating diffraction, meaning that all the cells (periods) of a straight guide participate to building-up a backward wave that cancels the incident one. If the periodicity of the waveguide is broken, e.g., by a bend, the coherence of the wavelets disappears, and transmission may even be, in such regions, stronger than in a straight waveguide. Hence, great care should be exerted before claiming high bend efficiencies as the structure does not act, powerwise, as the simple concatenation of straight and bent sections. Normalization is meaningful only in regions of sufficiently large transmission of the straight guide. Therefore, data such as the spectrum of the reference straight guide should in principle be systematically displayed to assess the validity of the comparison and to point out the anomalous low transmission regions of the straight waveguide (the ministopbands) where normalized data should be considered and interpreted with the greatest care.

\section{B. Influence of the Guide Width and of the PC Parameters}

Efforts in integrated optics have been mainly devoted to monomode waveguides. A monomode behavior is obtained for widths larger than $1 \mu \mathrm{m}$ (hence, easier lithography steps) only if the lateral index step $\Delta n$ (or effective index step) is sufficiently low. This, in turn, leads to important losses for bends with radii smaller than $100 \mu \mathrm{m}$ to $10 \mu \mathrm{m}$, depending on $\Delta n$. As the optical barriers are "perfect" for PC guides, any guide larger than the material's half wavelength, about $250 \mathrm{~nm}$, is basically multimodal. Even the single-missing row guide $\mathrm{W} 1$ is far from being systematically monomode. Hence, although multimodality is an unwanted feature, we have to cope with it if we want to use guides of acceptable size which lend themselves better to the various in- and outcoupling to conventional waveguides required in integrated optics. (a)

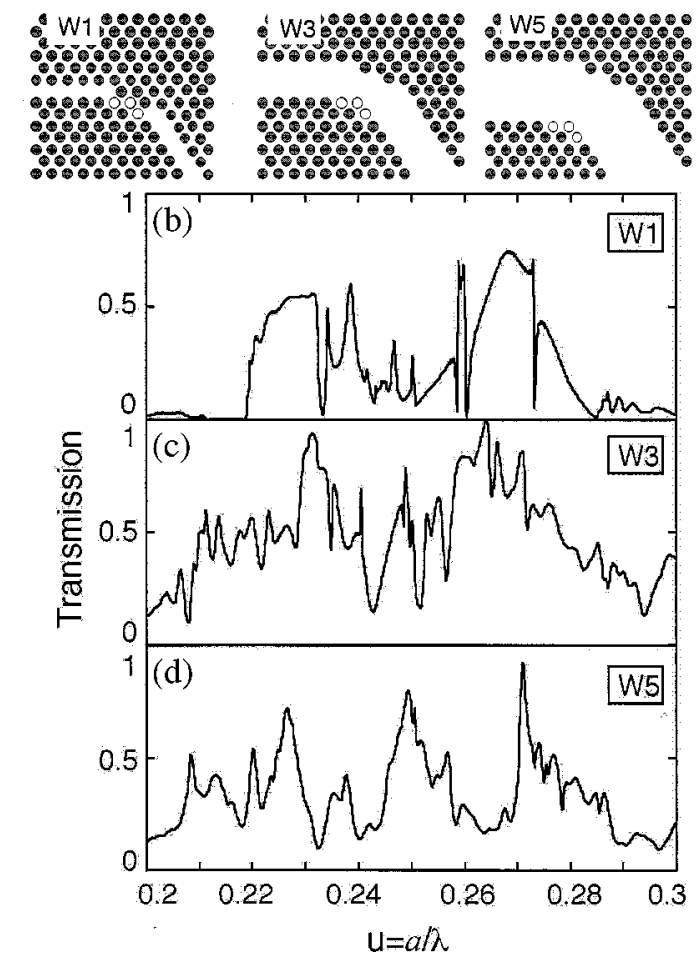

Fig. 2. Influence of guide width: (a) layout of the bends W1, W3, W5 smoothed by the displacement of three holes from the inner to the outer corner; associated simulated transmission spectra for the bend in: (b) W1; (c) W3; and (d) W5. Dielectric constant $\varepsilon=11.56$. Air-filling factor $f=0.35$. Arm lengths: see Table I for all calculations.

Let us examine the effect of guide width that is, of course, the primary parameter to affect the multimodality of the guide. Typically the number of modes allowed in a $\mathrm{W} n$ scales like $n$.

Fig. 2(b) shows the transmission level of various $60^{\circ}$ bends which have been "cut" by a $30^{\circ}$ section, which amounts to displacing three holes from the inner side to the outer side of the bend, as shown in Fig. 2(a). This bend design has been chosen in order to increase the average transmission and to show the generality of the transmission behavior versus guide width, irrespective of the particular bend design. The three widths selected here are $\mathrm{W} 1, \mathrm{~W} 3$, and $\mathrm{W} 5$; hence, the straight sections are symmetric with respect to their axis. The air filling factor is $f=35 \%$ and the matrix dielectric constant is $\varepsilon_{\text {eff }}=11.56$. The length of the arms is short $(10 \mathrm{a}, 8 \mathrm{a})$ in order to isolate the bend effect. One might fear that for W5, this is excessively short. However, our source directionality is such that we saw no substantial changes upon varying the arm length.

There are two important facts to note. The first point is that the degree of interferences ("speckle") increases as the guide width increases. This is in agreement with the fact that increasingly more modes are available and their beating becomes more complex. The waveguide $\mathrm{W} 1$ presents two monomode regions which correspond to the two flat, relatively high transmission regions in the spectrum (from $a / \lambda=0.220$ to 0.232 and from 0.262 to 0.272 ). Note that the absolute normalization of $\mathrm{W} 1$ data is particularly delicate as the result seems to have more dependence on the source profile; it might be that the "true" maximum transmission is larger in such a bend (see the membrane 


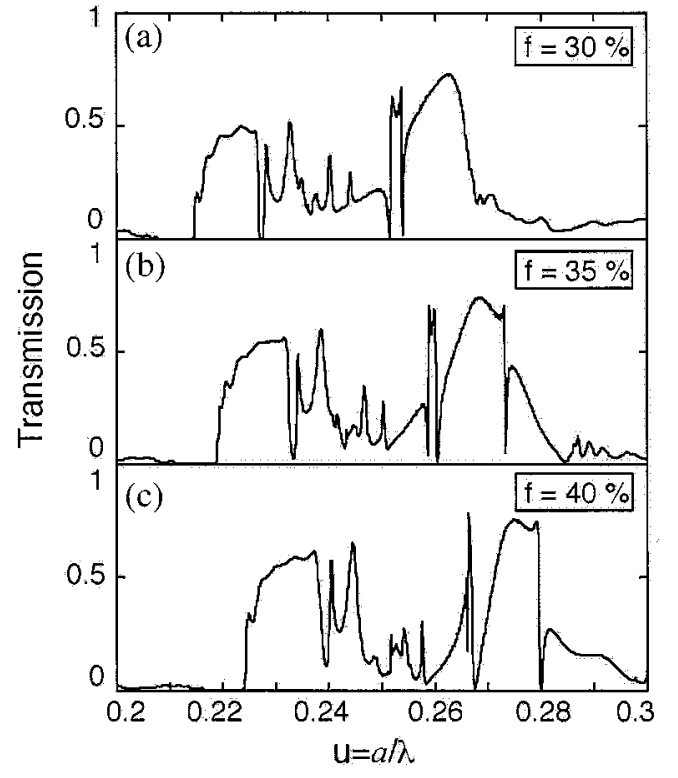

Fig. 3. Influence of PC air-filling factor $f$ : calculated transmission spectra of the bent waveguide W1 for: (a) $f=30 \%$; (b) $f=35 \%$; and (c) $f=40 \%$. Dielectric constant $\varepsilon=11.56$.

experiment of [13]), say up to 0.85 rather than 0.75 . The more disturbed part of the spectrum $(a / \lambda=0.23-0.26)$ corresponds to multimode regions, for which interferences associated with mode scrambling may occur. W3 and W5 are multimode in the frequency range considered: W3 supports 4 to 6 guided modes depending on the frequency and W5 supports 6 to 9 modes.

The second point is that there is no obvious winner among the three waveguides with the kind of transmission calculated here, based on an integrated Poynting vector measurement, not on modal transmission measurement. Note finally that, for the short bend "arms" used here (10 rows), the mini-stopbands of W3 and W5 are barely visible; e.g., for the fundamental mode of $\mathrm{W} 3$, it lies around $a / \lambda=0.252$, causing only weak troughs in the spectrum. One of the reasons, already discussed above, is the absence of coherence between the two straight sections, which minimizes this mode coupling phenomenon. The smoother transmission windows of the single-missing row W1 certainly make its use easier in a real system.

For us, it also has interest for easing further analysis, and in particular the analysis of the dependence of transmission spectra on the PC air filling factors $f$. Spectra for $\mathrm{W} 1$ and increasing air filling factors $f=30 \%, 35 \%, 40 \%$ are shown in Fig. 3, with the same matrix dielectric constant $\varepsilon_{\text {eff }}=11.56$. The smooth windows are seen to shift regularly to higher frequencies as $f$ increases. They also tend to slightly broaden. These trends closely follow the evolution of the guide dispersion relations, with allowed bands of the guide shifting to higher frequencies as the physical width of the guide is reduced by the larger hole radius. Another effect is the increase of the gap's high frequency edge, which also attracts some of the high-order guided modes while leaving the fundamental mode rather unaffected, and is the likely origin of the broadened transmission windows. Finally, the overall increase in transmission level with increasing $f$ can also be related to the stronger confinement: the narrower

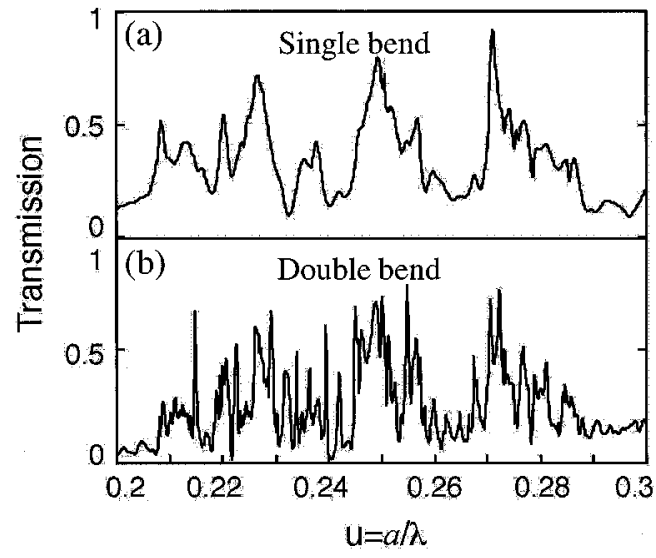

Fig. 4. (a) Single and (b) double-bend transmission spectra in a W5 waveguide. Dielectric constant $\varepsilon=11.56$. Air-filling factor $f=0.35$.

the mode profile, the better mode matching occurs at the bend, and the higher the transmission.

Until now, we have seen that spectra tend to be dominated by modal interference effects. These are also an issue when cascading bends. To assess the impact of cascading bends, we show in Fig. 4 the transmission spectrum $T_{1}$ of the W5 (broad) single-bend guide already shown in Fig. $2\left(f=35 \%, \varepsilon_{\text {eff }}=\right.$ 11.56) and compare it to the transmission spectrum $T_{2}$ of a double-bend (one bend to the left, the other to the right), with similar straight sections as in Fig. 2, and twenty PC rows between the two bends. It is seen that the double-bend waveguide transmission $T_{2}$ is rarely the square of the single-bend transmission $T_{1}^{2}$. Rather, it is much more wiggly than $T_{1}^{2}$, and occasionally larger, especially at some narrow spikes at $a / \lambda=0.215$, $0.240,0.255$, etc. We attribute these spikes to the resonant transmission through modes localized between the two bends (basically, modes of the in-between straight guide section that experience sufficiently reflective conditions at the two bends).

To conclude this first scan through the basic parameters (width, air filling-factor, number of bends in series), numerical data evidence a number of interferometric effects that disturb transmission, sometimes constructively. We will see below, however, that these effects tend to be washed out when losses are turned on, which is closer to the present experimental situation.

\section{Influence of the Bend Design}

A bend is a transition whereby modes from straight sections have no special reason to match to each other one by one. In the worst case, a bend is mainly a mode "scrambler" which could arbitrarily mix all the modes concerned, i.e., the ingoing fundamental mode with all waveguide modes propagating forward or backward (plus radiation losses discussed below, Section II-D). This means, of course, a lot of reflection in the input section (but not necessarily into the fundamental mode), and a poor transmission of the fundamental mode.

However, there are several reasons to hope that introducing a short section at $30^{\circ}$ in a $60^{\circ}$ bend can help a lot. This is also the idea implemented by Mekis for a $90^{\circ}$ bend in the pillar PBG system [2]. One idea is that the pair of transitions that occur can 
(a)
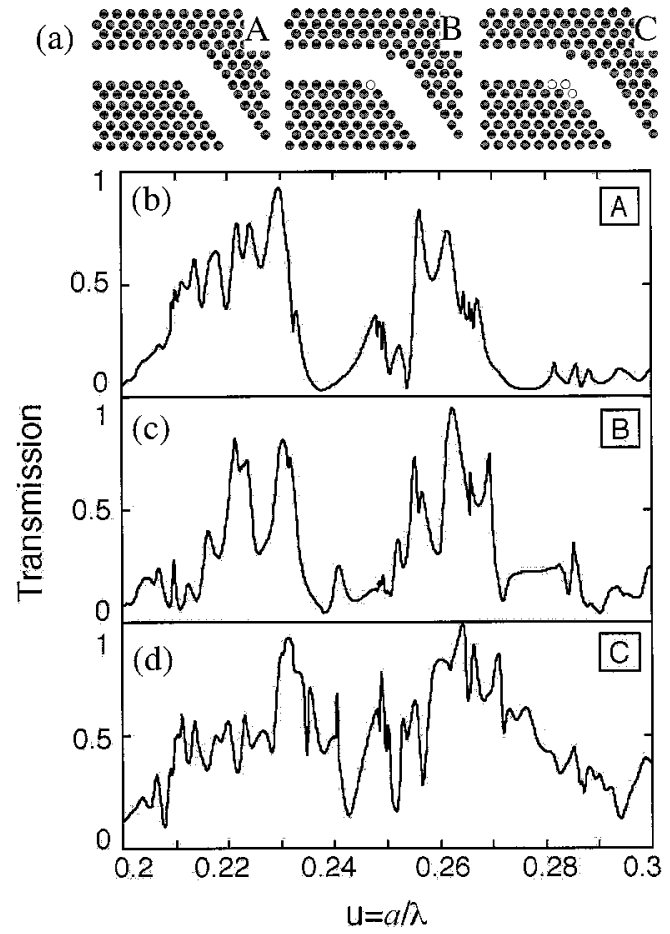

Fig. 5. (a) Bend designs A, B, C in W3. (b) Associated simulated transmission spectra for: (b) design A; (c) design B; and (d) design C. Dielectric constant $\varepsilon=$ 11.56; air-filling factor $f=0.35$.

interfere in such a way that reflections are destroyed, just as in a symmetric Fabry-Perot cavity at resonance (zero reflection). Another reason is that, in a naive ray picture, such a $30^{\circ}$ section behaves as a mirror that can exactly send, if properly located, a ray from one side to the other. Of course, the actual waves will not follow such a simple behavior given the large confinement, but the guideline is an interesting one and is obviously helpful to reduce mode scrambling: even if the reflection in a confined geometry partly spoils the mode profile, one may hope that the result is much better than what a random "scrambler" would provide. A last issue, as far as mode scrambling is concerned, is polarization mixing. This is not allowed in a 2-D picture of a 2-D-PC guide bend. But the actual 3-D nature of the problem is liable to induce such a mode conversion, some hints of which are discussed in the experimental section. Conversely, we can guess that our moderate vertical index confinement, which makes the 3 -D system quite closer to a 2-D system than membrane-based structures [38], [39], could be helpful in reducing this possible polarization mixing penalty.

Let us now attempt to smooth the bend since this simple option is supported by various points of views (interferences as in [2], the corner mirror limit). To this end, we focus on the W3 guide. Fig. 5(a) shows, in addition to the basic bend of design $\mathrm{A}$, two designs $\mathrm{B}$ and $\mathrm{C}$ corresponding to the displacement of one and three holes from the inner to the outer bend corner. Their respective transmissions, plotted in Fig. 5(b), show that this method is quite successful. In the low frequency regime $(a / \lambda=0.21-0.23)$ however, design $\mathrm{A}$ is performing well, sometimes better than B and $\mathrm{C}$. In the higher frequency range, smoothing has a clear effect on transmission, especially in the range $a / \lambda=0.265-0.30$. In the intermediate region,
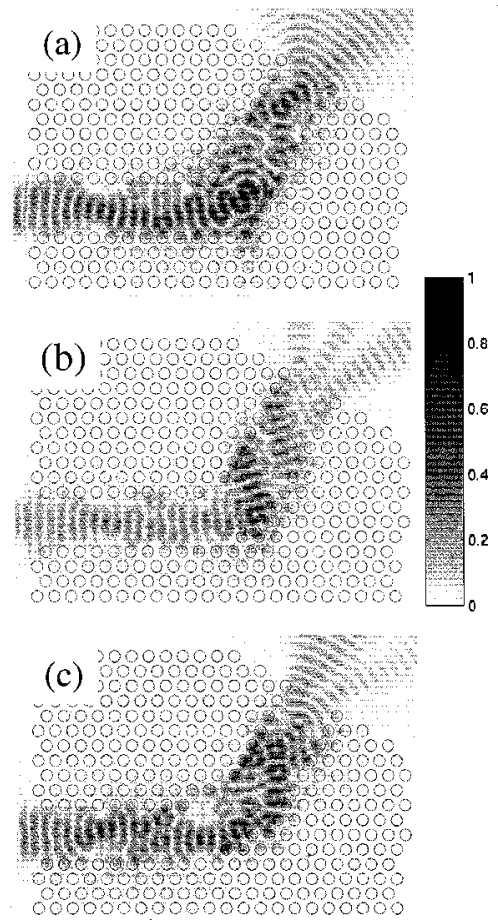

Fig. 6. Magnetic field patterns of $\mathrm{W} 3$ bends at the frequencies giving the best transmission level around $\alpha / \lambda=0.23$, i.e., (a) $u=0.2297$ for bend $\mathrm{A}$; (b) $u=0.2305$ for bend $\mathrm{B}$; and (c) $u=0.2312$ for bend $\mathrm{C}$ (parameters of Fig. 5).

around $a / \lambda=0.24$, only the smoothest bend $\mathrm{C}$ exhibits a sizable transmission, while bends $\mathrm{A}$ and $\mathrm{B}$ have both quite low transmissions.

To get further insight, an exhaustive analysis of field patterns would be needed. This would be lengthy as these patterns change in nature at each of the marked spectral features. We only concentrate here on the peak transmission around $a / \lambda=$ 0.23 , which seems common to all three designs. We display in Fig. 6(a)-(c) the intensity maps of the $\mathrm{H}$ field patterns as obtained for excitation at the neighbor peak frequencies $(a / \lambda=$ 0.2297 for design $\mathrm{A}, a / \lambda=0.2305$ for design $\mathrm{B}, a / \lambda=0.2312$ for design $C$ ). On the input side (the horizontal segment), the straight wave fronts of the incoming field are perturbed by the reflected fields. This effect seems minor only in design B but the output field for design B clearly exhibits three lobes, suggesting that the fundamental mode has only a weak contribution to the transmission in this case. Conversely, the radiated output field patterns of designs $\mathrm{A}$ and $\mathrm{C}$ are closer to that of the fundamental mode. At this stage, it thus seems that the transmission peak of design $\mathrm{B}$ around $a / \lambda=0.2305$ is due to a combination of minimal reflection, and favored coupling to a high-order mode. Finally, can we find from the field pattern a plausible explanation for the transmission peak of design A, the canonical W3 bend? The only striking feature is the more important field concentration right at the bend corner, compared to the other cases. This suggests that a weak resonant-cavity effect takes place at the corner. Bends based on open cavities have been investigated in the case of deep-etched ridge waveguides [41], and it could be that even the canonical $60^{\circ}$ bend design A exhibits a similar behavior. Light localization at bends of PC guides is still an open matter, to our knowledge [42]. 
We also investigated the effect of smoothing on other guide widths, especially W5, and we found that the smoothing effect is rather systematically positive. The directions to pursue are of two kinds: first, more smoothing, e.g., by displacing more than three holes, in order to look for some optimal behavior and second, variants of the simple hole displacement from inner to outer corner, such as addition or deletion of holes, sublattice-step displacements of some holes, etc. To establish an optimum, some composite figure of merit is needed, taking into account, e.g., the best transmission and a $-3 \mathrm{~dB}$ transmission window of reasonable spectral width (e.g., $30 \mathrm{~nm}$ at $1550 \mathrm{~nm}$, which corresponds to only 0.005 around 0.5 in units of $a / \lambda$ ) together with an acceptable degree of ripple within this window. Such studies are in progress in our group.

\section{Influence of the Out-of-Plane Losses}

Losses out of the plane are liable to occur in our system as it retains a high index cladding, with the consequence that the PBG range always lies above the cladding light line. But in this configuration, one can obtain a proper estimate of losses and show that they scale as $(\Delta \varepsilon)^{2}$. This was shown in [25] as a result of considering the separation of variables in the propagation equation, using a scalar wave analysis. As an important additional result, it was shown that one can then cast the out-of-plane radiation losses into an imaginary dielectric constant $\varepsilon^{\prime \prime}$ in the air holes. More refined studies since then have confirmed various aspects of this assumption [37], [43]. Here we use the same method to account for the effect of 3-D losses in bends. Bends are, indeed, a case for which the assumption that there is no coherence between the dipole emitters associated with the perturbation approach of [25] is more justified than in plain crystals or in straight waveguides: the specific 2-D distribution of the field calculated above translates into a complicated pattern of emitters that have little chance to give rise to strong coherence effects.

We exemplify the use of the imaginary dielectric constant in the case of the W3 bent waveguide smoothed according to design $\mathrm{C}$. The value $\varepsilon^{\prime \prime}=0.2$ was selected in agreement with [25], although the different heterostructure and the different etch depth might affect this value in either direction. Fig. 7(a) shows as a solid line the new spectrum obtained with this level of losses, and, for comparison, the lossless spectrum (dashed line). The most striking effects are the smoothing and lowering of the transmission values, a trend already observed in the case of straight waveguides [37]. Here the two relative maxima, $T=$ 0.9 to 0.99 for the lossless case at $a / \lambda=0.231$ and 0.264 , respectively, fall to $T=0.6$ and 0.55 .

To understand what happens, it is instructive to remark that the marked minima of the lossless case are almost unaffected in absolute value, with some hints that the lossy case might even transmit more than the lossless one, at $\alpha / \lambda=0.209$ and 0.293 . This counter-intuitive effect finds a natural explanation if we remind that interferences between excited modes are responsible for the peaks and troughs: at peaks, there are constructive interferences, the field that builds up is large, and, as in a Fabry-Perot cavity, any losses act directly on the amplitudes of interfering modes, and prevent the field to build up properly. The power that has built up is strongly radiated away at the bend (dissi-
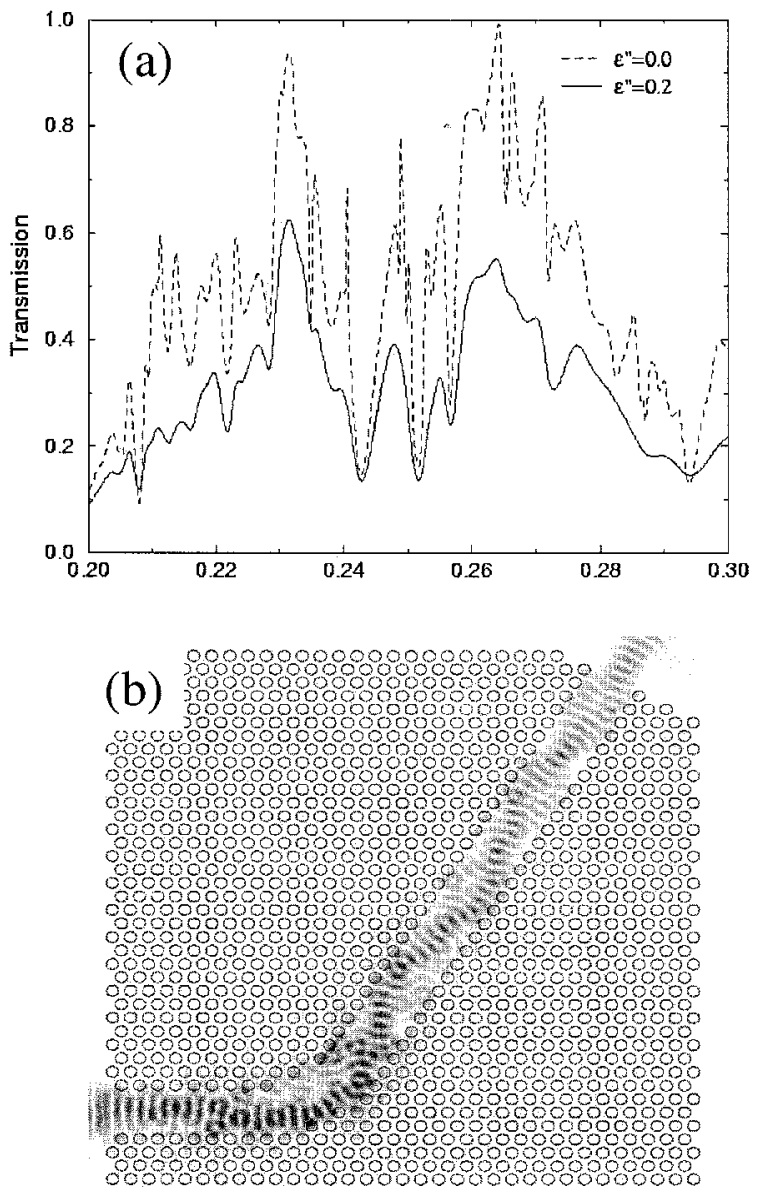

Fig. 7. (a) Bend transmission without (dashed line) and with (solid line) losses. Dielectric constant $\varepsilon=11.56$; air-filling factor $f=0.35$. Here, the radiation losses in the third dimension are accounted for in a 2-D calculation through a dissipative medium of dielectric constant $1+0.2 i$ in the air holes; (b) Typical field pattern with a long exit waveguide showing the faster decay of high order modes generated at the bend compared to the fundamental mode.

pated in our 2-D ansatz) and depletes the transmitted channel. At troughs, there are destructive interferences, which are, in turn, attenuated by the losses. One of the main intervening modes has less loss than the other(s) and the outgoing field may benefit from the amplitude of this mode without too much cancellation by the others. This leads, in the present case, to a small net effect, for example at $\alpha / \lambda=0.242,0.252,0.258$. This behavior is similar to, e.g., that of the symmetric Fabry-Perot cavity, with zero reflection at resonance only in the lossless case, and nonzero reflection in case of losses.

For multimode guides, an interesting aspect of dealing with a slightly lossy PC pertains to the different propagation losses encountered by the various modes: while the fundamental mode has weak overlap with the PC holes and, hence, weak propagation losses, the high order modes have more overlap, about a factor of ten higher if we compare the third to the fundamental mode, and hence, more losses. The underlying differences in relative field-PC overlap are sizable, about a factor of ten between the third and the fundamental mode of W3. Fig. 7(b) shows the natural modal filtering effect that arises when a long exit waveguide is implemented after the bend, here with a loss parameter $\varepsilon^{\prime \prime}=0.22$ similar to Fig. 7(a): the darker ghost ray that appears after the bend and makes then one or two zigzags 


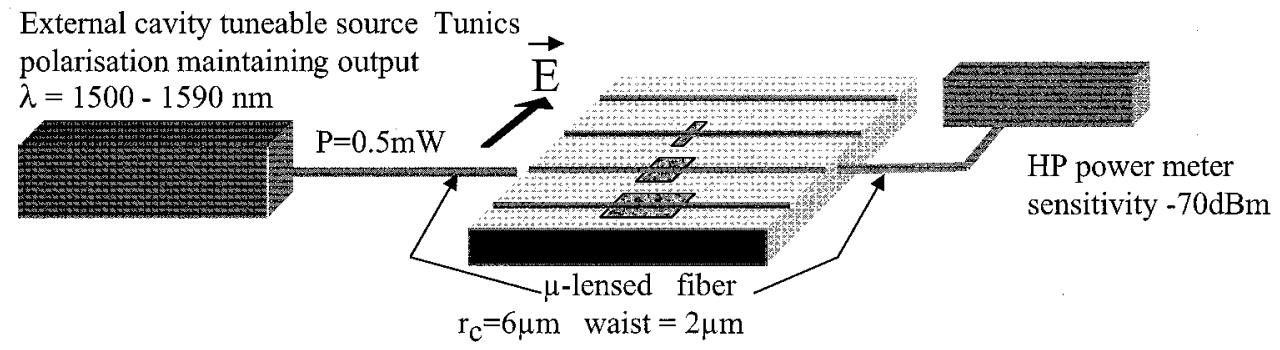

(a)

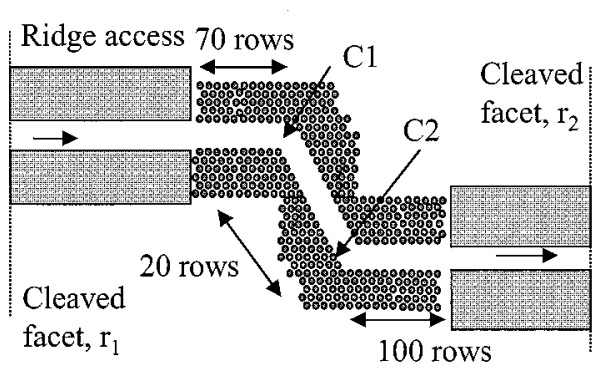

(b)

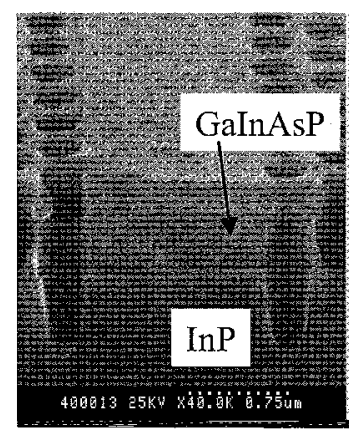

(c)

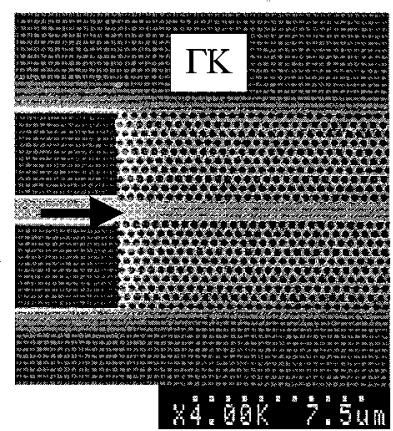

(d)

Fig. 8. (a) Fiber-to-fiber measurement setup; (b) Layout of a typical double-bend structure; (c) Micrograph of a PC waveguide cross section; (d) Micrograph of the ridge to $\mathrm{PC}$ guide transition.

is a signature of the sizable amplitude of the third mode generated at the bend. As propagation proceeds, however, the pattern is purified from this "ray," and only the fundamental mode remains. This suggests a simple way to perform a modal projection to extract the transmission of the fundamental mode only. Such a projection would, indeed, help fitting better the experimental data as the experimental collection scheme favors the fundamental mode, as will be seen in the next section. However, this approach has a sizable computational cost, and cannot discriminate pairs of modes whose inverse of absorption difference, $(\Delta \alpha)^{-1}$, is smaller than the extra length added $L$.

To conclude these theoretical studies, it is seen that out-ofplane losses should be included before an optimal design can definitely be selected. All the likely ingredients of a bend's figure of merit (peak $T,-3 \mathrm{~dB}$ bandwidth, ripple) depend directly on the loss parameter. Nevertheless, we have checked that the general trends of lossless studies, such as the increasing merit of designs A, B, C, were similar with the introduction of losses, at least up to values of $\varepsilon^{\prime \prime}=0.2-0.4$. A refined study showed that the transmission of bend design A was much more affected by the introduction of losses than bend designs $\mathrm{B}$ and C. This can be understood when considering that bend design A has a more "resonant-like" behavior than the others and thus is more sensitive to losses.

\section{ChARACTERIZATION}

\section{A. End-Fire Method}

We start this experimental section with the end-fire method applied to InP-based systems, which is popular in integrated optics. Here, transmission measurements are performed using a coaxial fiber-to-fiber bench in TE polarization [Fig. 8(a)], unless stated otherwise. A constant optical power $P=0.5 \mathrm{~mW}$
$(-3 \mathrm{dBm})$ is launched from a tunable source $(1500-1590 \mathrm{~nm})$ and collected through microlensed fibers. In order to ensure a reproducible coupling in and out of a PC bent waveguide, the latter is inserted between two uniform monomode-TE ridge access guides. We have measured various double-bend waveguides like in Fig. 8(b). Together with each bent waveguide, an adjacent PC straight guide of equal length was fabricated. The $\mathrm{PC}$ parameters (period $a=450 \mathrm{~nm}$, air-filling factor $f=45 \%-50 \%$ ) allow the operating wavelength $\lambda=1.55 \mu \mathrm{m}$ to fall within the photonic bandgap.

The confinement in the vertical direction is provided through total internal reflection in a gas-source molecular beam epitaxy (GSMBE) GaInAsP layer, 500-nm-thick, which has a photoluminescence peak at $1.22 \mu \mathrm{m}$, and is capped by a $200-\mathrm{nm}$-thick InP protecting layer. The 2-D PC is dry-etched in a methanebased plasma, the etch depth is limited to $1.1 \mu \mathrm{m}$. Channel waveguides such as W2 and W3 are formed by removing 2 or 3 rows along the $\Gamma K$ direction. Micrographs of the cross section and of a ridge-PC transition are shown in Fig. 8(c) and (d), respectively.

We have investigated in some depth the W3 double-bend waveguides for all three designs A (no hole displaced), B (1 hole displaced), and C (3 holes displaced) underlined above. The calculated transmission spectra for designs $\mathrm{B}$ and $\mathrm{C}$ are presented in Fig. 9(a) (they result from a fit, see below), and the measured ones are shown in Fig. 9(b), together with the transmission spectrum of a reference straight waveguide. Transmission spectra of straight and bent guides are presented independently rather than ratioing them as already justified. The double-bend waveguide with no hole displaced (design A) had poor transmission (about $-70 \mathrm{dBm}$ optical power) and is not plotted. The coupling efficiency between the ridge access guide and the straight PC guide was previously measured to 

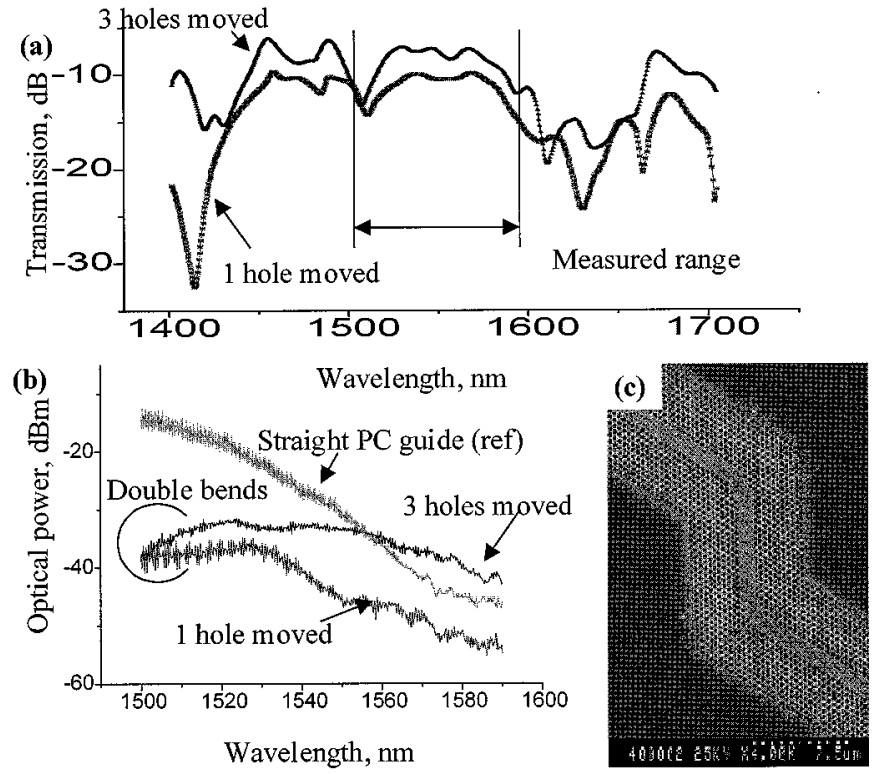

Fig. 9. (a) Calculated transmission spectra for designs $\mathrm{B}$ and $\mathrm{C}$, in $\mathrm{dB}$, as a function of wavelength for the fitted parameters (period $a=450 \mathrm{~nm}, f=$ $50 \%, \varepsilon^{\prime \prime}=0.22$ ). (b) Measured optical power spectra of W3 double bends for designs $\mathrm{B}$ and $\mathrm{C}$, compared to the straight waveguide (top) when a power of $-3 \mathrm{dBm}(0.5 \mathrm{~mW})$ is launched at the source. (c) Micrograph of a double-bend waveguide with $\mathrm{C}$ design.

be better than $95 \%$, in agreement with the above simulations of Section II-A and the measurements of [14]. The straight PC guide spectrum exhibits a dip (ministopband) at large wavelengths that we could attribute to a mode coupling mechanism of the fundamental mode with a high order mode [6]. Clearly, displacing 1 and 3 holes in the corner (designs B and C) greatly improves the measured transmission, in agreement with the above FDTD simulations.

In the present case [Fig. 9(a)], a fit has been attempted with the air-filling fraction and the imaginary dielectric constant $\varepsilon^{\prime \prime}$ as the adjustable parameters, the index value being adequate for the present heterostructure, $\varepsilon_{\text {eff }}=10.56$. The best fit of the measured spectra is obtained for an air-filling factor of $50 \%$, and an imaginary part of the dielectric constant $\varepsilon^{\prime \prime}=0.22$. Obviously, these figures point to rather high losses that we attribute to the limited etch depth [25]. The moderate remaining discrepancies between the theory and experiments could stem from the filtering out of high-order modes by the output ridge [see Section II-D above and Fig. 7(b) for the effect of losses on this filtering out].

Reflections at a bend are one of the possible drawbacks of PC bends. We show here that our fiber-to-fiber setup may give a quantitative estimate of the bend reflection. To this end, we exploit the narrow fringes that one can guess on the experimental spectra of Fig. 9(b). These fringes are associated with cavities. It is well known that a cavity of length $L$ and index $n$ with reflectors $r_{1}$ and $r_{2}$ gives rise to a modulated transmission $T(\lambda)$ :

$$
T(\lambda)=\left(\frac{1}{1+\left(r_{1} r_{2}\right)^{2}+2 r_{1} r_{2} \cos (4 \pi n L / \lambda)}\right)^{2} .
$$

To visualize easily the adequate fringes in spite of the smooth variations of the bend transmission, we first formed a smoothed
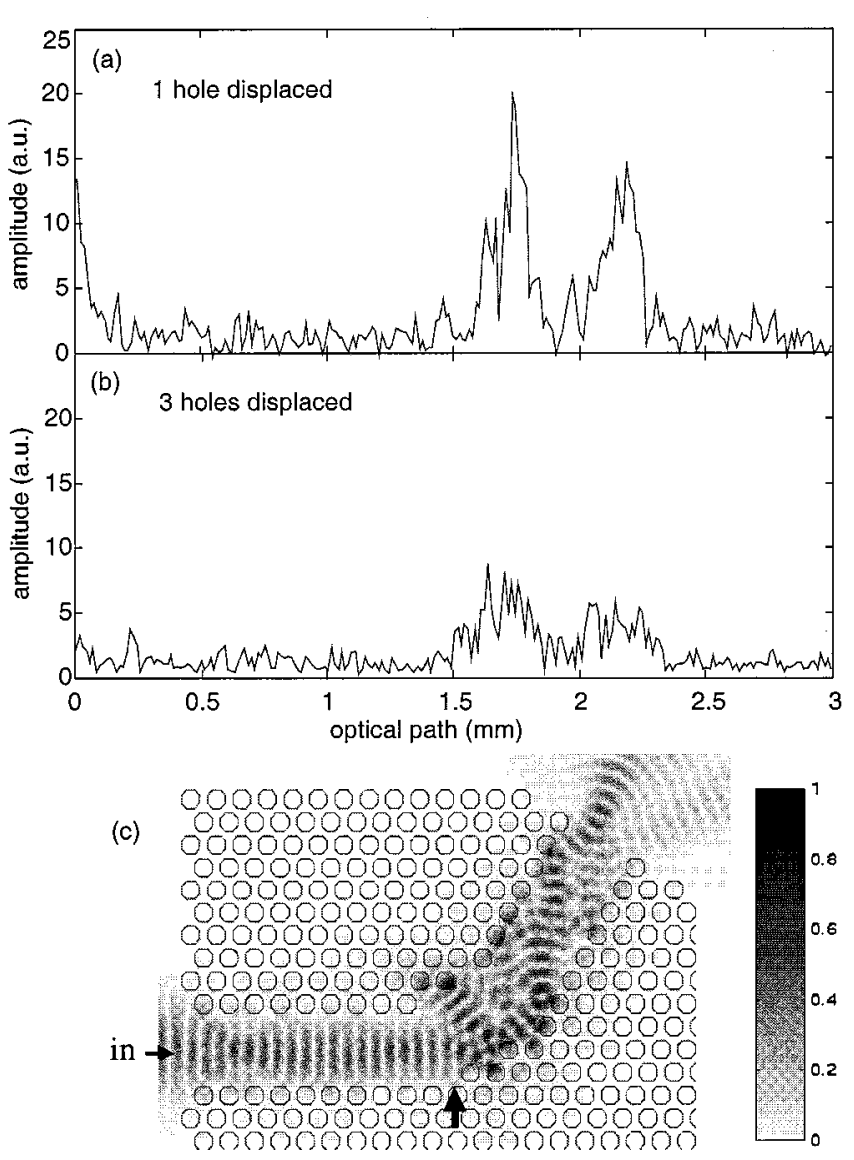

Fig. 10. Analysis of reflections at $\mathrm{W} 3$ bends with $\mathrm{B}$ and $\mathrm{C}$ designs. (a), (b) Fourier transformed spectra for the $\mathrm{B}$ and $\mathrm{C}$ designs in which the peak positions are directly related to cavity lengths. (c) Field pattern calculated around the bend region, showing that the incoming field is not affected by reflected field in the input branch.

"low-pass-filtered" intensity spectrum $\langle I(1 / \lambda)\rangle$ and then obtained the "normalized" fringes by using $I(1 / \lambda) /\langle I(1 / \lambda)\rangle$. The filter characteristic was flat up to a "frequency" $\Delta \lambda^{-1}=$ $1 /(7 \mathrm{~nm})$ and decreased linearly to zero for a "frequency" $\Delta \lambda^{-1}=1 /(5 \mathrm{~nm})$ to apodize its response. Then, to find the relevant cavities, we use the Fourier transform of the spectrum $I(1 / \lambda) /\langle I(1 / \lambda)\rangle$, and obtain strong amplitudes whenever a characteristic oscillation period exists in the spectrum. Examples of such Fourier transformed data for the B and $\mathrm{C}$ bend designs are given in Fig. 10(a) and (b), respectively, directly scaled in terms of the cavities optical paths $L_{\mathrm{opt}}$. In our case, the two peaks seen in Fig. 10(a) and (b), similar in strength, are associated with the cavities defined between the input facet of the sample $\mathrm{r} 1$ and the first bend corner $\mathrm{C} 1$, and similarly between the second bend corner $\mathrm{C} 2$ and the output facet $\mathrm{r} 2$. This can be safely deduced from the knowledge of the group effective index ( $n_{\text {group }}$ is about 3.6): $L_{\text {opt }} / n_{\text {group }}$ corresponds to the physical length $L_{\text {phys }}$ of the straight ridge + PC sections, as measured under a microscope. The transmission spectra are then Fourier-filtered by standard signal processing techniques to remove all but the desired cavities and the fringe contrast $v$ of the resulting spectrum (the ratio of maximum to minimum transmitted intensities) is measured. The reflectivities $r_{1}$ and $r_{2}$ of the facets are known independently from a previous 
calibration, as well as the propagation losses of the ridge $\left(\alpha_{r}\right)$ and of the straight PC waveguide $\left(\alpha_{P C}\right)$ sections (lengths $L_{r}$ and $L_{\mathrm{PC}}$ ), using straight guides without and with PC sections. The $\mathrm{C} 1$ corner reflectivity $r_{C 1}$ is then obtained according to the formula:

$$
L n r_{1} r_{C 1}-\alpha_{r} L_{r}-\alpha_{P C} L_{\mathrm{PC}}=\operatorname{Ln} \frac{1-\sqrt{\nu}}{1+\sqrt{\nu}} .
$$

We assume that $r_{C 1}$ is not wavelength dependent, which is reasonable as the wavelength range used for the measurements is small in comparison with the whole PBG range. For design $\mathrm{B}$, the bend reflection is $r_{C 1}=0.495$, and it is considerably reduced to $r_{C 1}=0.126$ for design $\mathrm{C}$. This drop of the reflection for the smoothest design $\mathrm{C}$ is also visible on the field pattern shown in Fig. 10(c) calculated with a filling factor of 50\%, and at the frequency $a / \lambda=0.2812$, corresponding to the range of interest. Unlike the patterns shown earlier, the wave at the entrance of the bend is here quite unperturbed. Still, at the corner, there is quite some mode conversion toward high-order modes, which is also partly responsible for the washing out of the mini-stopband, clearly visible on the measured transmission of Fig. 9(b), where the spectrum for design $\mathrm{C}$ does not drop at long wavelengths.

Turning now to the influence of the PC guide width, we measured the transmission level through W3 and the narrower W2 double-bend waveguides. The comparison of the transmission levels of the straight reference W3 waveguide and the double-bend $\mathrm{W} 3$ waveguide at $1540 \mathrm{~nm}$ (i.e., outside the mini-stopband region), leads to a value of losses of $3 \mathrm{~dB} / \mathrm{bend}$ for the best $\mathrm{C}$ design. Similar W2 double-bend waveguides have been fabricated and measured for all three designs A, $\mathrm{B}$, C, with the same PC period $a=450 \mathrm{~nm}$ and a similar filling-factor and etch depth. Again the best transmission level is obtained for design $\mathrm{C}$, but now with losses of $2.25 \mathrm{~dB} / \mathrm{bend}$ at $1580 \mathrm{~nm}$ (again outside the mini-stopband of the straight W2 waveguide). This trend is compatible with Fig. 2 above (one must take care of the poorer ridge-PC coupling in the case of $\mathrm{W} 2$, which is not a symmetric waveguide), and with the general "mode scrambling" argument.

Let us finally address briefly the polarization issue: unlike the symmetries which may heavily restrain polarization conversion in straight PC guides, bent PC guides (and bent ridge guides as well) are likely to exhibit sizable polarization conversion. Of course, this effect cannot be modeled easily in two dimensions, only in three dimensions. Hence the experimental approach is quite welcome in tackling this issue. To detect polarization conversion, a polarizer is inserted between the collecting microlensed fiber and the photodiode. A TE mode is launched from the source and propagated within the ridge access guide. Both TE and TM intensities are collected at the exit of the double-bend waveguide. In the case of W2, no significant result was observed. In the case of W3, we clearly see on Fig. 11 a TM spectrum having the same spectral behavior as the TE one, with a difference of about $12 \mathrm{~dB}$, which means a signal significantly larger than the residual TM polarization of the source. This is a first indication of polarization mode conversion occurring in the double-bend waveguide. Further studies are needed to assign the origin of this conversion in the cascaded bends, in relation to the multimodal behavior of each polarization.

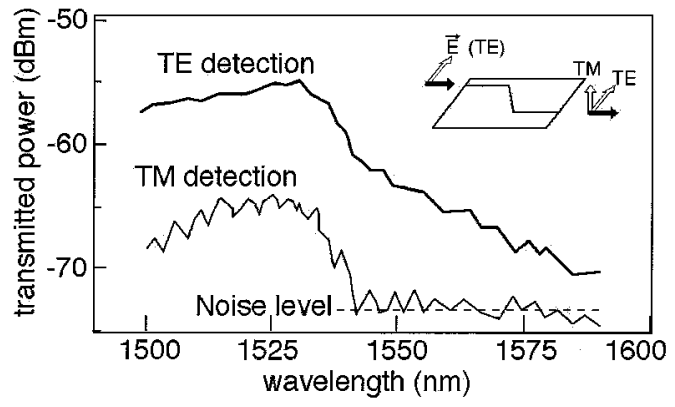

Fig. 11. Spectra of a W3 double-bend waveguide similar to Fig. 8 obtained for TE excitation and either TE or TM collection, showing indications of polarization conversion.

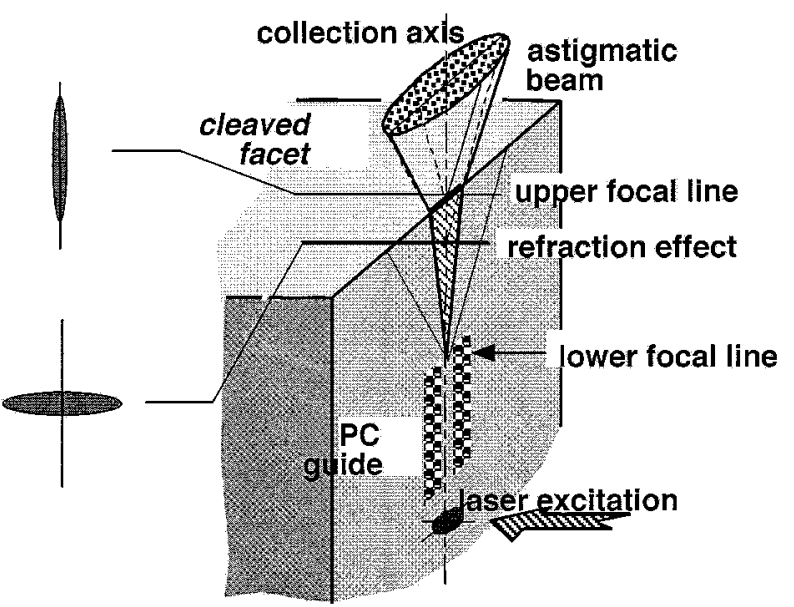

Fig. 12. Principle of the internal probe method for waveguide measurements.

\section{B. Internal Probe Method}

We examine here the application of the internal probe method, described in [44], to the measurement of single and double bends. Note that this experimental method is essentially reproduced in the numerical configuration without access ridges illustrated in Fig. 1(a). In brief, one excites, by means of a focused laser spot, the photoluminescence (PL) of species such as InAs quantum dots, or InGaAsP quantum wells, embedded in the heterostructure of interest. The guided part of the PL is then collected at a cleaved edge, after interaction with the PC pattern under study. A reference signal may be measured in a nearby unpatterned area with excitation at the same distance of the cleaved edge to remove the guided mode reabsorption and thus obtain quantitative measurements. Two of the main advantages of this method are its reproducibility (the coupling uncertainties of the end-fire method, that require great care, are eliminated) and its flexibility. It allows, for example, the examination of single and double bends etched on the same sample without any difficulty. One of the delicate issues in the application of the method to PC guides is to unambiguously collect light that has traveled through the guides. The method used in [22], whereby an in-depth focus is used to directly visualize the guide exit inside the wafer, is a good way to lift such ambiguities: we illustrate in Fig. 12 the fate of guided PL collected by the microscope objective. The collected beam is astigmatic, and one of its focus lies at the exit of the PC guide. Light emerging from the PC guide thus appears as an elongated 
segment when using this focus. Spatially selective collection coupled to the camera then ensures that only the relevant beams are collected, with minimal crosstalk.

We used here a GaAlAs guiding heterostructure (similar to that of [22], with essentially a thick $\mathrm{Ga}_{0.2} \mathrm{Al}_{0.8}$ As bottom cladding, a 260-nm-thick GaAs core, and a 300-nm-thick $\mathrm{Ga}_{0.8} \mathrm{Al}_{0.2}$ As top cladding), which comprises in its core three layers of embedded InAs quantum dots with a deliberately inhomogeneous size and spectrum distribution in order to have a broadband PL internal source, as well as modest reabsorption. The spectral InAs QD's distribution is peaked around $1080 \mathrm{~nm}$ and gently vanishes around $1190 \mathrm{~nm}$, which gives access to a large spectral range in contrast with the end-fire method where the tunable laser is limited to less than $100 \mathrm{~nm}$ spectral range.

We start with the measurements of single W3 bends using a spectrometer with a silicon CCD detector that limits the spectral range of the source to $950-1060 \mathrm{~nm}$. In order to circumvent this limitation and to extend the normalized frequency range $u=a / \lambda$ probed experimentally, similar patterns of identical filling factor $f=35 \%$ were fabricated for two periods $a=240$ and $a=260 \mathrm{~nm}$ ("lithographic tuning"), allowing the normalized frequency range $u=0.23-0.28$ to be covered. Some data treatment is then needed to compensate for the index dispersion and to take full advantage of the lithographic tuning when superimposing spectra from different periods on a common $u=a / \lambda$ axis hence, at different $\lambda$. The material dispersion results in an effective index dispersion of the guided mode $\partial n / \partial \lambda=$ $-7.810^{-4} \mathrm{~nm}^{-1}$. This dispersion tends to compress and shift the spectra with respect to those that would result from fixed refractive index. All the data were corrected for this dispersion using the relation $\lambda=\lambda_{1}+\partial \lambda / \partial n \times\left(n-n_{1}\right), \lambda=1000 \mathrm{~nm}$ being the wavelength for which $n_{1}^{2}=\varepsilon_{1}=11$. With this correction, the spectra from $a=240$ and $a=260 \mathrm{~nm}$ can be safely superimposed.

The overall length of the bent waveguides was 60 rows and the three different bend designs A, B, C were investigated (no hole, one hole and three holes displaced from the inner to the outer corner).

We normalized the bent waveguide spectra by a reference guided-PL spectrum in an unpatterned area near each kind of bent waveguide and at the same distance of the cleaved edge. The purpose of this normalization is to remove the spectral shape of the PL and to eliminate possible PL yield variations (usually less than 5\% throughout such small areas) but we note that this method does not yield an absolute transmission. The spectra corresponding to the three designs A, B, C are displayed in Fig. 13(a)-(c) as a function of the normalized frequency on the same arbitrary intensity scale, allowing a direct comparison of the relative transmission level among the different bend designs.

The major spectral feature in the transmission of the simple bend [design A, Fig. 13(a)], besides small intensity variations upon frequency, is a dip at $u=0.26$, which is on account of the transmission ministopband of the straight waveguide sections. Note the good agreement between the spectra provided by both periods in the region of their frequency overlap. For the bend smoothed through the displacement of one hole from the inner to the outer corner [design B, Fig. 13(b)], the overall transmission
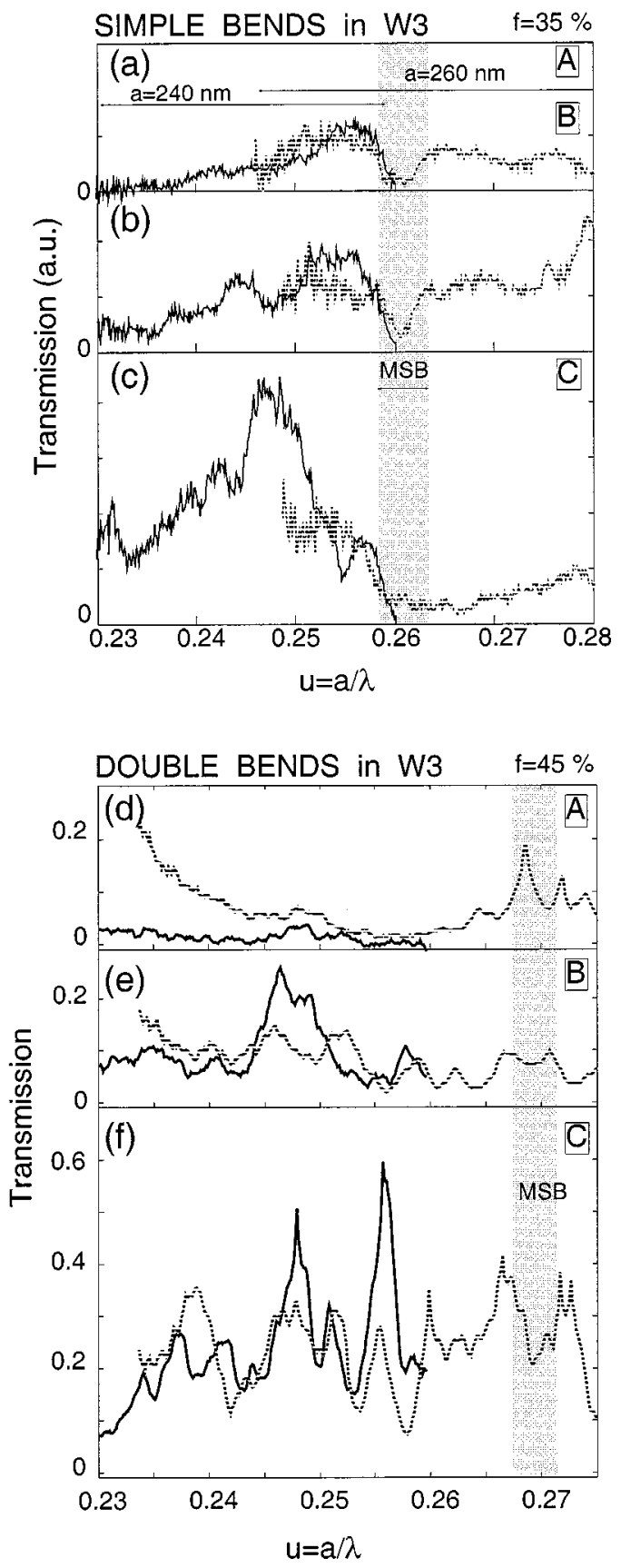

Fig. 13. Internal probe method on a GaAs-based samples, with InAs QDs as PL sources: (a)-(c) Transmission spectra through single bends in W3 for the designs A, B, C; two periods $240 \mathrm{~nm}$ (solid line) and $260 \mathrm{~nm}$ (dotted line) were measured using a Si detector, the filling factor is $f=35 \%$; (d)-(f) Transmission spectra through double bends in W3 normalized by the transmission of a reference straight waveguide of the same length for designs A, B, C. Two periods $240 \mathrm{~nm}$ (solid line) and $260 \mathrm{~nm}$ (dotted line) were measured using an InGaAs detector. The filling factor is $f=50 \%$. For each set of samples (single and double bends), the frequency range of the ministopband of the straight reference waveguide is indicated in gray.

level is improved by a factor of 2 . For the last design with three holes displaced [design C, Fig. 13(c)], the transmission is further improved by a factor of 2 for the period $240 \mathrm{~nm}$ (the signal for the period $260 \mathrm{~nm}$ is lower due to a damaged cleaved facet at this sample location). These results clearly show that smoothing the bend greatly improves the overall transmission level, in agreement with theoretical predictions presented above in Section II. 
When comparing these spectra to the theoretical ones in Fig. 5, it appears that the spectral features are much less pronounced in the experimental case due to the presence of slight out-of-plane losses.

Double bends in W3 were probed using a similar experimental setup. At variance with the previous measurements of single bends, the excitation laser is an argon laser. Also, to extend the measured spectrum with respect to the previous case of a Si-based detector, an InGaAs photodiode array with cutoff around $1600 \mathrm{~nm}$ was used after the spectrometer. This second setup was validated against the one used above.

We investigated the losses per bend in W3 double-bend waveguides for the three bend designs A, B, C by comparison with reference straight waveguides of identical width $\mathrm{W} 3$ and length (60 rows). The intermediate section between the two bends was 15 rows long. Two different PC periods 240 and $260 \mathrm{~nm}$ were fabricated, and the filling factor was kept constant at $50 \%$.

In a first step, we normalized both the straight and bent waveguide data by a reference guided-PL spectrum to correct the spectral shape of the PL as previously. In a second step, the transmission of the bent guide was normalized with respect to the straight reference guide. As explained earlier, care must be taken of the ministopband regions in the transmission spectra upon normalization. For the W3 guide fabricated with $f=50 \%$, the ministopband is now centered around $u=0.27$.

Fig. 13(d)-(f) shows the normalized transmission data. The trend, in agreement with the above simulations, can be clearly observed: there is an overall pronounced increase in transmitted light intensity going from bend design A to C. Regarding the detail of the spectral features, they are sharper than for simple bends due to the Fabry-Perot oscillations between the two bends (see Fig. 4 for the modeling of this effect). For the smoothest bend design $\mathrm{C}$, a maximal normalized transmission as high as 0.6 could be measured at $u=0.256$ for the period $240 \mathrm{~nm}$, which corresponds to a loss of $1.1 \mathrm{~dB} / \mathrm{bend}$, which points to losses weaker than those introduced in Fig. 7, for which one would drop to about $2 \mathrm{~dB}$ per bend. The uncertainty here is on the order of $0.25 \mathrm{~dB}$. Of course, on a broader wavelength range of 15-20 nm, losses are typically $1 \mathrm{~dB}$ higher. The transmission of the "sharper" bends (designs A and B) is approximately a factor of two lower, with losses as high as 7.6 and $3.0 \mathrm{~dB} /$ bend with a smoother spectral behavior (about 40-50 nm $1 \mathrm{~dB}$ bandwidth).

Note that small fluctuations in the fabrication process can have a significant influence on the transmission spectral features, as transmission through bent waveguides is sensitive to the position of single holes at the bend, and also to their depth, which is not exactly the same for different periods of the PC crystal. This could be the main origin of the discrepancies in transmission levels in the region of spectral overlap of different periods [Fig. 13(d)-(f)]. The interesting feature, however, is the very good overall similarity of the spectral shape despite the differences in transmission level.

Both methods, end-fire in InP systems and internal probe in GaAs systems, enable the measurement of the transmission of the fundamental mode through straight and bent waveguides. They lead to the same conclusion that the bend transmission is increased by inserting a small $30^{\circ}$ cut at the bend. One advantage of the internal probe method is the better reproducibility of the measurements and, hence, its better accuracy. The difficulty of the reproducibility of in- and out-coupling from fibers to access ridges is suppressed, and there is no more transition ridge-PC waveguide that slightly degrades the signal-to-noise ratio of the spectra, especially for waveguides narrower than W2. However, the absolute signal is much higher with a laser as used in the end-fire method, a factor which is in favor of the end-fire methods for narrow guides or system that have intrinsically low transmission. The internal probe method is also more flexible as it allows the measurement through a single-bend as well as through a double-bend waveguide, whereas in the configuration of the end-fire method, the input and output ridges must be oriented along the same axis normal to the cleaved facets of the sample, allowing the measurement of double-bend waveguides only. The measurement of a single bend waveguide is important as it was pointed out in the theoretical section II. Namely, the transmission through double-bend waveguides is not necessarily the square of the transmission through single-bend waveguides, as constructive interferences may occur between the two bends and possibly reconvert high order modes excited at the first bend back into the fundamental mode at the second bend. However, the major advantage of the end-fire method is the possibility of estimating the reflection coefficient of one bend.

\section{DISCUSSION AND CONCLUSION}

We have seen that the performance of PC bent guides is still not satisfying, with reported loss levels between $1.1 \mathrm{~dB} /$ bend and $3 \mathrm{~dB} /$ bend for the various cases seen earlier. These values are in reasonable agreement with theoretical predictions. However, one of the main uncertainties in comparing theory and experiment is the role of high order modes: both measurement methods used here tend to filter out the fundamental mode quite efficiently, whereas simulations include all outgoing modes, fundamental and higher.

Hence, a first direction towards better understanding is to compare modal transmission to overall transmission, with a similar work on reflections.

A second direction is to find out which part of a bend insertion loss comes intrinsically from the design, and what part is added by the out-of-plane radiation losses. This can be substantiated by simulations with and without the adjunction of $\varepsilon^{\prime \prime}$ in the air holes. On the experimental side, one could compare bends defined in PCs with variable etch depths. The possibility to shift to a resonant bend would be valid only if the present level of impact of out-of-plane radiation losses is weak; otherwise, it is obvious that, as a resonance forces the wave to sample better the $\mathrm{PC}$, increased losses and poorer performance will result, especially at the resonant frequency.

Once these issues are properly addressed, or simultaneously, what are the perspectives for improving the performance of the existing designs? In addition to the cavity-bend just signaled above, we can find two roads. The first road is to further investigate the approach of a modified light path in the vicinity of the bend, by using different kinds of bend smoothing, or 
by changing the local width of the guide, or by a combination of both. Bend smoothing leads, of course, to a larger effective bend size but the photonic crystal bends still remain compact compared to classical bends in integrated optics and they have a larger operating bandwidth. This road essentially applies to bends based on "broader" PC guides, say W2, W3, or even larger, that are largely multimode but present the advantage of being less lossy than the narrower W1 guide as the mode confinement is larger and therefore, the overlap of the field with the air holes is smaller. The second road concerns the narrower W1 guide that has the advantage of supporting a reduced number of modes (down to the monomode limit in the proper spectral regions), which should make the bend design easier: for this system, a hole is a big perturbation to the basic bend, so that one should use more continuous bends adjustments. These could be locally larger or smaller holes if the etching allows, and subperiod shifts in hole position, in the spirit of the cavity tuning methods proposed by Painter [39] for cavities.

We have seen that a combination of intensive modeling, integrated optics ideas and accurate experimental measurements is a good mix for addressing the many issues underlying the proper design of efficient PC bends. We believe that a continued effort with these three ingredients will bring the performance to a competitive level and make PC bends a viable compact alternative to present, bulky integrated optics solutions.

\section{REFERENCES}

[1] J. D. Joannopoulos, R. D. Meade, and J. N. Winn, Photonic Crystals, Molding the Flow of Light. Princeton, NJ: Princeton Univ. Press, 1995.

[2] A. Mekis, J. C. Chen, I. Kurland, P. R. Villeneuve, and J. D. Joannopoulos, "High transmission through sharp bends in photonic crystals waveguides," Phys. Rev. Lett., vol. 77, pp. 3787-3790, 1996.

[3] A. Chutinan and S. Noda, "Waveguides and waveguide bends in two-dimensional photonic crystal slabs," Phys. Rev. B, vol. 62, pp. 4488-4492, 2000.

[4] T. Baba, N. Fukaya, and J. Yonekura, "Observation of light propagation in photonic crystal optical waveguides with bends," Electron. Lett., vol. 35, pp. 654-655, 1999.

[5] T. Baba, N. Fukaya, and A. Motegi, "Clear correspondence between theoretical and experimental light propagation characteristics in photonic crystal waveguides," Electron. Lett., vol. 37, pp. 761-762, 2001.

[6] S. W. Leonard, H. M. V. Driel, A. Birner, U. Gösele, and P. R. Villeneuve, "Single-mode transmission in two-dimensional maroporous silicon photonic crystal waveguides," Opt. Lett., vol. 25, pp. 1550-1552, 2000.

[7] S. Olivier, M. Rattier, H. Benisty, C. J. M. Smith, R. M. D. L. Rue, T. F. Krauss, U. Oesterle, R. Houdré, and C. Weisbuch, "Mini-stopbands of a one dimensional system: The channel waveguide in a two-dimensional photonic crystal," Phys. Rev. B, vol. 63, pp. 113-311, 2001.

[8] S. Olivier, H. Benisty, C. J. M. Smith, M. Rattier, C. Weisbuch, and T. F. Krauss, "Transmission properties of two-dimensional photonic crystal channel waveguides," Opt. Quantum Electron., 2001, to be published.

[9] M. Swillo, M. Qiu, M. Mulot, B. Jaskorzynska, S. Anand, A. Talneau, and A. Karlsson, "Characterization and modeling of InP/InGaAsP photonic crystal waveguides," in Proc. ECOC, 2001.

[10] —, "Transmission spectra and loss estimation in InP-based photonic crystal waveguides," Appl. Phys. Lett., submitted for publication.

[11] M. Tokushima, H. Kosaka, A. Tomita, and H. Yamada, "Lightwave propagation through a $120^{\circ}$ sharply bent single-line-defect photonic crystal waveguide," Appl. Phys. Lett., vol. 76, pp. 952-954, 2000.

[12] M. Loncar, D. Nedeljkovic, T. Doll, J. Vuckovic, A. Sherrer, and T Pearsall, "Waveguiding in planar photonic crystals," Appl. Phys. Lett., vol. 77, pp. 1937-1939, 2000

[13] E. Chow, S. Y. Lon, J. R. Wendt, S. G. Johnson, and J. D. Joannopoulos, "Quantitative analysis of bending efficiency in photonic crystal waveguide bends at $\lambda=1.55 \mu \mathrm{m}$ wavelengths," Opt. Lett., vol. 26, pp. 286-288, 2001.
[14] A. Talneau, L. L. Gouezigou, and N. Bouadma, "Quantitative measurements of low propagation losses at $1.55 \mu \mathrm{m}$ on planar photonic crystal waveguides," Opt. Lett., vol. 26, pp. 1259-1261, 2001.

[15] J. Moosburger, M. Kamp, and A. Forchel, "Transmission spectra measurements on photonic crystals based bent waveguides," in Proc. of CLEO, Baltimore, May 6-11, 2001.

[16] Y. Sugimoto, N. Ikeda, N. Carlsson, K. Asakawa, N. Kawai, and K. Inoue, "Simulation, fabrication and characterization of $2 \mathrm{D}$ photonic crystal slab with defect waveguides for PLC applications," in Proc. of PECS III, St. Andrews, U.K., June 9-14, 2001.

[17] W. Bogaerts, P. Bienstman, D. Taillaert, R. Baets, and D. De Zutter, "Out-of-plane scattering in photonic crystal slabs," IEEE Photon. Tech. Lett., vol. 13, pp. 565-567, 2001.

[18] D. Labilloy, H. Benisty, C. Weisbuch, T. F. Krauss, R. M. De La Rue, V Bardinal, R. Houdré, U. Oesterle, D. Cassagne, and C. Jouanin, "Quantitative measurement of transmission, reflection and diffraction of two-dimensional photonic bandgap structures at near-infrared wavelengths," Phys. Rev. Lett., vol. 79, pp. 4147-4150, 1997.

[19] D. Labilloy, H. Benisty, C. Weisbuch, C. J. M. Smith, T. F. Krauss, R. Houdré, and U. Oesterle, "Finely resolved transmission spectra and band structure of two-dimensional photonic crystals using InAs quantum dots emission," Phys. Rev., vol. B59, pp. 1649-1652, 1999.

[20] H. Benisty, C. Weisbuch, D. Labilloy, M. Rattier, C. J. M. Smith, T. F. Krauss, R. M. De La Rue, R. Houdré, U. Oesterle, and D. Cassagne, "Optical and confinement properties of two-dimensional photonic crystals," J. Lightwave Technol., vol. 17, pp. 2063-2077, 1999.

[21] S. Olivier, C. Smith, M. Rattier, H. Benisty, C. Weisbuch, T. Krauss, R. Houdré, and U. Oesterlé, "Miniband transmission in a photonic crystal coupled-resonator optical waveguide," Opt. Lett., vol. 26, pp. 1019-1021, 2001

[22] C. J. M. Smith, H. Benisty, S. Olivier, M. Rattier, C. Weisbuch, T. F. Krauss, R. M. De La Rue, R. Houdré, and U. Oesterle, "Low-loss channel waveguides with two-dimensional photonic crystal boundaries," Appl. Phys. Lett., vol. 77, pp. 2813-2815, 2000.

[23] C. J. M. Smith, T. F. Krauss, H. Benisty, M. Rattier, C. Weisbuch, U. Oesterle, and R. Houdré, "Directionally dependent confinement in photonic-crystal microcavities," J. Opt. Soc. Amer. B, vol. 17, pp. 2043-2051, 2000.

[24] C. J. M. Smith, R. M. De La Rue, M. Rattier, S. Olivier, H. Benisty, C. Weisbuch, T. F. Krauss, R. Houdré, and U. Oesterle, "Coupled guide and cavity in a two-dimensional photonic crystal," Appl. Phys. Lett., vol. 78 , pp. 1487-1489, 2001.

[25] H. Benisty, D. Labilloy, C. Weisbuch, C. J. M. Smith, T. F. Krauss, A. Béraud, D. Cassagne, and C. Jouanin, "Radiation losses of waveguidebased two-dimensional photonic crystals: Positive role of the substrate," Appl. Phys. Lett., vol. 76, pp. 532-534, 2000.

[26] P. Lalanne and H. Benisty, "Ultimate limits of two-dimensional photonic crystals etched through waveguides: An electromagnetic analysis," $J$. Appl. Phys., vol. 89, pp. 1512-1514, 2001.

[27] H. Benisty, P. Lalanne, S. Olivier, M. Rattier, C. Weisbuch, C. J. M. Smith, T. F. Krauss, C. Jouanin, and D. Cassagne, "Finite-depth and intrinsic losses in vertically etched two-dimensional photonic crystals," Opt. Quantum Electron., to be published.

[28] H. Benisty, "Modal analysis of optical guides with two-dimensional photonic band-gap boundaries," J. Appl. Phys., vol. 79, pp. 7483-7492, 1996.

[29] J. B. Pendry, "Transfer matrix techniques for electromagnetic waves," in Photonic Band Gap Materials, C. M. Soukoulis, Ed. Dordrecht: Kluwer, 1996, p. 203

[30] K. Sakoda, M. Sasada, T. Fukushima, A. Yamanaka, N. Kawai, and K. Inoue, "Detailed analysis of transmission spectra and Bragg reflection spectra of a two-dimensional photonic crystal with a lattice constant of $1.15 \mu \mathrm{m}$," J. Opt. Soc. Amer. B, vol. 16, pp. 361-365, 1999.

[31] M. Qiu, K. Azizi, A. Karlsson, M. Swillo, and B. Jaskorzynska, "Numerical studies of mode-gaps and incoupling efficiency for line-defect waveguides in two-dimensional photonic crystals," Phys. Rev. B, Sept. 15,2001

[32] S. Fan, J. N. Winn, A. Devenyi, J. C. Chen, R. Meade, and J. D. Joannopoulos, "Guided and defect modes in periodic dielectric waveguides," J. Opt. Soc. Amer. B, vol. 12, pp. 1267-1272, 1995.

[33] M. Qiu and S. He, "A nonorthogonal finite-difference time-domain method for computing the band structure of a two-dimensional photonic crystal with dielectric and metallic inclusions," J. Appl. Phys., vol. 87, pp. 8268-8275, 2000.

[34] _ "Numerical method for computing defect modes in two-dimensional photonic crystals with dielectric or metallic inclusions," Phys. Rev. B, vol. 61, pp. 12871-12876, 2000 . 
[35] — " "Guided modes in a two-dimensional metallic photonic crystal waveguide," Phys. Lett. A, no. 266, pp. 425-429, 2000.

[36] J. P. Berenger, "Perfectly-matched layers boundary conditions," $J$. Comput. Phys., pp. 185-200, 1994

[37] M. Qiu, B. Jaskorzynska, M. Swillo, and H. Benisty, "Time-domain 2D modeling of slab-waveguide based photonic-crystal devices in the presence of out-of-plane radiation losses," J. Lightwave Technol., submitted for publication.

[38] B. D'Urso, O. Painter, J. O’Brien, T. Tombrello, A. Yariv, and A. Scherer, "Modal reflectivity in finite-depth two-dimensional photonic crystal microcavities," J. Opt. Soc. Amer. B, vol. 15, pp. 1155-1159, 1998.

[39] O. Painter, J. Vuckovic, and A. Scherer, "Defect modes of a two-dimensional crystal in an optically thin dielectric slab," J. Opt. Soc. Amer. B, vol. 16, pp. 275-285, 1999.

[40] C. Manolatou, S. G. Johnson, S. Fan, P. R. Villeneuve, H. A. Haus, and J. D. Joannopoulos, "High-density integrated optics," J. Lightwave Technol., vol. 17, pp. 1682-1692, 1999.

[41] R. L. Espinola, R. U. Ahmad, F. Pizzuto, M. J. Steel, and R. M. Osgood, "A study of high-index-contrast $90^{\circ}$ waveguide bend structures," Opt. Express, vol. 8, pp. 517-528, 2001.

[42] A. Mekis, S. Fan, and J. D. Joannopoulos, "Bound states in photonic crystal waveguides and waveguide bends," Phys. Rev. B, vol. 58, pp. 4809-4817, 1998.

[43] H. Benisty, S. Olivier, M. Rattier, and C. Weisbuch, "Applications of two-dimensional photonic crystals to semiconductor optoelectronic devices," in Photonic Crystal and Light Localization in the 21st Century, C. M. Soukoulis, Ed. Dordrecht, The Netherlands: Kluwer, 2001

[44] D. Labilloy, H. Benisty, C. Weisbuch, T. F. Krauss, R. Houdré, and U. Oesterle, "Use of guided spontaneous emission of a semiconductor to probe the optical properties of two-dimensional photonic crystals," Appl. Phys. Lett., vol. 71, pp. 738-740, 1997.

Henri Benisty received the Ph.D. degree (with a dissertation on accumulation layers at Si interfaces) from Ecole Normale Supérieure, Paris, in 1989.

Since then, his research topics have been first nanostructure growth and physics (Thomson, Orsay, France) and lamellar III-VI compounds (Paris VI University). Since 1994, he has been involved with the Laboratoire de Physique de la Matière Condensée, Ecole Polytechnique, Palaiseau, France, working on research on planar cavities (mainly for LEDs) and in photonic crystals in two dimensions on III-V, with both experimental and theoretical approaches. He currently investigates applications of photonic crystals to LEDs and to miniature photonic integrated circuits.

Ségolène Olivier graduated from the Ecole Supérieure de Physique et Chimie, Paris, France, in 1999. She is currently working toward the Ph.D. degree in the Photonic Crystal Group at the Laboratoire de Physique de la Matiere Condensee, Ecole Polytechnique, Palaiseau, France.

Her research interests include photonic crystal structures for miniature photonic integrated circuits.

\section{Claude Weisbuch was born in 1945.}

From 1969 to 1979, he was an Assistant Professor with the University of Paris specializing in solid-state physics. He joined Bell Laboratories, Murray Hill, NJ, as a Member of Technical Staff from 1979 to 1981, working on the optical properties of quantum wells. From 1981 to 1983 , he was with the Compagnie de Saint-Gobain as a "Directeur de Recherches" in charge of Exploratory Research. He was the Director of the Physics Laboratory in the Central Research Laboratories of Thomson-CSF from 1983 to 1992 and an Associate Professor of Physics at the Ecole Polytechnique from 1984 to 1996. Since September 1992 he has been with the Laboratoire de Physique de la Matière Condensée, Ecole Polytechnique, Palaiseau, France, working as a Directeur De Recherches, Centre National de la Recherche Scientifique (CNRS). He has authored or co-authored more than 140 papers, five books, and holds ten patents. He co-directed five international schools or workshops on physics. His research activities involve optics of semiconductors and the physics and applications of low-dimensional structures such as quantum-well or quantum dot lasers. His most recent research is on the intriguing fundamental properties and applications of coupled semiconductors and optical fields in microcavities and photonic crystals.

Prof. Weisbuch is a member of the Academy des Technologies and a Fellow of the American Physical Society.
Mario Agio was born in 1975, in Piacenza, Italy. He received the diploma (laurea) in physics from the University of Pavia, Pavia, Italy, in 1999, where he is currently working toward the Ph.D. degree in physics in conjunction with Iowa State University, Ames, through the Memorandum of Agreement "International Doctorate."

He was a Research Scholar at Ames Laboratory DOE, Ames, IA, from September to December 1999. He is also an Associate to INFM and to Ames Laboratory, DOE. His research activities concern the theory of photonic crystals.

Maria Kafesaki received the Ph.D. degree in 1997 from the Physics Department, University of Crete, Crete, Greece.

She is a Postdoctoral Researcher with the Theoretical and Computational Division of the IESL-FORTH. She has worked as a Postdoctoral Researcher in the Consejo Superior de Investigaciones Scientificas, Madrid, Spain, and in IESL of FORTH. Her research is mainly in the area of electromagnetic, acoustic, and elastic wave propagation in periodic and random media, where she has long-time theoretical and computational experience. She has 15 publications in refereed journals and conference proceedings.

Costas M. Soukoulis received the B.S. degree from the University of Athens, Athens, Greece, in 1974, and the M.S. and Ph.D. degrees in physics from the University of Chicago, Chicago, IL, in 1975 and 1978, respectively.

He has been a Faculty Member of the Physics Department, Iowa State University, Ames, since 1984. He was a Visiting Assistant Professor at the University of Virginia from 1978 to 1981 and a Research Physicist at Exxon Research and Engineering Company from 1981 to 1984. Since 1983, he has had a strong collaboration with the Research Center of Crete, Heraklion, Crete, Greece. His current research interests include theory of disordered systems, light localization, photonic crystals, random lasers, and left-handed materials.

Min Qiu (M'99) received the Ph.D. degree in condense matter physics from Zhejiang University, Hangzhou, China, in 1999, and the second Ph.D. degree in electromagnetic theory from the Royal Institute of Technology (KTH), Stockholm, Sweden, in 2001.

From 1999 to 2000, he was a Visiting Scientist with the Department of Electromagnetic Theory, Royal Institute of Technology (KTH). He joined the Department of Microelectronics and Information Technology, at the same university, in 2001, where he is currently an Assistant Professor with the Laboratory of Optics, Photonics and Quantum Electronics. He has published more than 30 international refereed journal papers since 1996. His research interests include photonic crystals, integrated optical circuits, solid-state theory, electromagnetic theory, and numerical techniques in electromagnetics.

Dr. Qiu is a member of the Optical Society of America (OSA).

Marcin Swillo received the M.Sc. degree in optoelectronics in 1993 and Ph.D. degree in physics in 1998, both from the Warsaw University of Technology, Warsaw, Poland.

In 1998, he joined the Laboratory of Photonics and Microwave Engineering, Royal Institute of Technology, Kista, Sweden. He is currently engaged in research on the photonic crystal devices.

Anders Karlsson received the M.Sc. degree in engineering physics in 1987 and the Ph.D. degree in electrical engineering in 1992.

$\mathrm{He}$ is a Professor of Quantum Photonics with the Department of Microelectronics and Information Technology, Royal Institute of Technology (KTH), Stockholm, Sweden. He has been a Visiting Researcher with the NTT Basic Research Laboratory, Nihon University, Stanford University, and a Lecturer at École Polytechnique, Palaiseau. His research interests are quantum information, quantum optics, photonic crystals, and vertical cavity lasers. He is the coordinator of the IST FET QuComm project, chairman of the COST 268 Action "Wavelength Scale Photonic Components in Telecommunications," and has been/is active in the ACTS VERTICAL, TMR "Microlasers and Cavity QED," and the IST PCIC "Photonic Crystal Integrated Circuits" projects. 
Bozena Jaskorzynska received the M.Sc. degree in solid-state physics from Warsaw University, Warsaw, Poland, in 1970, and the Ph.D. degree from the Institute of the Fundamental Technological Problems, Polish Academy of Sciences, in 1980, where she carried out research in the field of diffraction and microwave theory.

After a Dutch government Postdoctoral fellowship with the Delft University of Technology, Delft, The Netherlands, during 1982-1983, where she dealt with laser physics, she was with the Institute of Optical Research in Stockholm, Sweden, during 1984-1995. There she dealt with second-harmonic generation, impact of nonlinearities on fiber transmission, and she led the first project on Er-doped fiber amplifiers in Sweden. She is currently a Professor with the Microelectronics and Information Technology, Royal Institute of Technology, Sweden. Her current research interests include nonlinear fiber optics, integrated photonic circuits, optical amplification, and photonic crystals.

Anne Talneau received the agrégation de physique in 1984 and the Ph.D. degree in 1990 from Thomson LCR (Orsay, France). Her dissertation was on monomode DFB lasers.

She then joined the France Telecom research center (CNET), Bagneux, France, where she was in charge of research activity on new monolithic multiwavelength sources for WDM applications. In 1999, she moved to the French public research, CNRS, at LPN, Marcoussis, France, where she currently investigates the interest of photonic crystals for integrated optics, in particular in the framework of national and European projects.

Jürgen Moosburger studied physics and mathematics at the University of Wuerzburg, Wuerzburg, Germany. He is now working as a Ph.D. student in the group of Alfred Forchel. His dissertation involves the development of fabrication techniques for photonic crystal based patterns on compound semiconductor heterostructures.

He also investigates and characterizes photonic crystal waveguide-based patterns.

Martin Kamp was born in Lippstadt, Germany, in 1971. He studied physics at the University of Würzburg, Würzburg, Germany, and spent one year at Stony Brook University, NY, where he received the M. A. degree in 1995. His thesis work was on semiconductor lasers with lateral feedback structures.

Currently, he is in charge of the Nanodevice Group of the Microstructure Laboratory, Würzburg. He is author or co-author of more than 30 papers relating to semiconductor lasers or photonic crystals structures. His current research interests include the development of new patterning technologies for semiconductor lasers, complex coupled DFB lasers, photonic crystals, and their application to optoelectronics.
Alfred Forchel received the Ph.D. degree in physics from Stuttgart University, Stuttgart, Germany, in 1982 with a dissertation on optical investigations of highly excited semiconductors. In 1988, he received the Dr. Habil. degree for studies on dimensionality dependent electronic properties of semiconductor structures.

From 1983 to 1989, he was in charge of the planning of the microstructure laboratory at Stuttgart. In 1990, he became a Full Professor of Physics at Würzburg University, Würzburg, Germany. In addition to the Chair of Technical Physics, he is responsible for the Microstructure Laboratory of Würzburg University. He is author or co-author of more than 300 papers relating mainly to optoelectronic devices and to optical properties of semiconductor microstructures. Currently, his main scientific interests are related to the development of novel III-V semiconductor structures for optoelectronic devices and the optical investigation of low-dimensional photonic and electronic structures.

Rolando Ferrini received the Diploma degree in physics in 1996 and the Ph.D degree (with a dissertation in condensed matter physics on optical properties of III-V semiconductors), both from the University of Pavia, Pavia, Italy,

From 1991 to 1995, he with Almo Collegio Borromeo, Pavia, Italy. In March 2000, he joined the Institut de Photonique et d'Electronique Quantique, Ecole Polytechnique Fédérale de Lausanne, Switzerland, where he is Scientific Collaborator in the Semiconductor Device Physics group. He is presently in charge of the group effort on photonic bandgap structures within the framework of the EEC-funded project Photonic Crystal Integrated Circuits (PCIC). His current research interests include advanced semiconductor optoelectronic components, in particular, 2-D photonic crystal structures and their applications to integrated optics. He is the author of more than 20 publications.

Romuald Houdré received the Ph.D. degree (with a dissertation on the photoemission from quantum wells and superlattices under negative electron affinity) from the Ecole Polytechnique, France, in 1985.

He spent one year as a Postdoctoral Fellow with the University of Illinois at Urbana-Champaign with Prof. H. Morkoc in the Molecular Beam Epitaxy (MBE) Group. He joined Ecole Polytechnique Fédérale de Lausanne (EPFL) in 1988 , where he is presently in charge of the optical microcavity and photonic crystal activity. His interest includes microcavities, photonic bandgap structures and molecular beam epitaxy. He is the author of 207 publications.

Ursula Oesterle graduated from the Department of Natural Sciences, Federal Institute of Technology, Zurich, Switzerland, and received the Ph.D. degree (with a dissertation on the growth and characterization of novel semiconductor vertical cavity emitters) from Ecole Polytechnique Fédérale de Lausanne (EPFL), Lausanne, Switzerland, in 1994.

Since 1994, she has been a scientific collaborator in charge of the Molecular Beam Epitaxy Crystal Growth Group at EPFL. She has also been the Program Manager for the Swiss Priority Program in Optics. She is currently with Swisscom, Switzerland, working on corporate technology. 